\title{
Inhibition of mitophagy drives macrophage activation and antibacterial defense during sepsis
}

\author{
Danish Patoli,, ${ }^{1,2}$ Franck Mignotte, ${ }^{1,2}$ Valérie Deckert, ${ }^{1,2}$ Alois Dusuel, ${ }^{1,2}$ Adélie Dumont, ${ }^{1,2}$ Aurélie Rieu, ${ }^{3}$ Antoine Jalil,, ${ }^{1,2}$ \\ Kevin Van Dongen, ${ }^{1,2}$ Thibaut Bourgeois, ${ }^{1,2}$ Thomas Gautier, ${ }^{1,2}$ Charlène Magnani, ${ }^{1,2}$ Naig Le Guern, ${ }^{1,2}$ Stéphane Mandard, ${ }^{1,2}$ \\ Jean Bastin, ${ }^{4}$ Fatima Djouadi, ${ }^{4}$ Christine Schaeffer, ${ }^{5}$ Nina Guillaumot, ${ }^{5}$ Michel Narce, ${ }^{1,2}$ Maxime Nguyen, ${ }^{1,2,6}$ Julien Guy, \\ Auguste Dargent, ${ }^{1,2,8}$ Jean-Pierre Quenot,, ${ }^{1,2,8,9}$ Mickaël Rialland, ${ }^{1,2}$ David Masson, ${ }^{1,2,10}$ Johan Auwerx, ${ }^{11}$ Laurent Lagrost, ${ }^{1,2,10}$ \\ and Charles Thomas ${ }^{1,2}$
}

¿Université de Bourgogne Franche-Comté (UBFC), UMR 1231, INSERM/AgroSup Dijon/Université de Bourgogne, Dijon, France. LipSTIC LabEx, Dijon, France. ${ }^{3}$ UBFC, UMR PAM A 02.102, AgroSup Dijon/ Université de Bourgogne, Dijon, France. ${ }^{4}$ Centre de Recherche des Cordeliers, INSERM, Université Sorbonne-Paris-Cité, Paris, France. ${ }^{5}$ Université de Strasbourg, CNRS, UMR 7178, LSMBO, Strasbourg, France. ${ }^{6}$ Department of Anesthesiology and Intensive Care, ${ }^{7}$ Hematology Laboratory, and ${ }^{8}$ Department of Intensive Care, University Hospital François Mitterrand, Dijon, France. ${ }^{9}$ Clinical Epidemiology, INSERM CIC 1432 and University of Burgundy, Dijon, France. ${ }^{10}$ Clinical Biochemistry Department, University Hospital François Mitterrand, Dijon, France. "'Laboratory for Integrative Systems Physiology, Institute of Bioengineering, Ecole Polytechnique Fédérale de Lausanne, Lausanne, Switzerland.

\begin{abstract}
Mitochondria have emerged as key actors of innate and adaptive immunity. Mitophagy has a pivotal role in cell homeostasis, but its contribution to macrophage functions and host defense remains to be delineated. Here, we showed that lipopolysaccharide (LPS) in combination with IFN- $\gamma$ inhibited PINIK1-dependent mitophagy in macrophages through a STAT1dependent activation of the inflammatory caspases 1 and 11. In addition, we demonstrated that the inhibition of mitophagy triggered classical macrophage activation in a mitochondrial ROS-dependent manner. In a murine model of polymicrobial infection (cecal ligature and puncture), adoptive transfer of Pink1-deficient bone marrow or pharmacological inhibition of mitophagy promoted macrophage activation, which favored bactericidal clearance and led to a better survival rate. Reciprocally, mitochondrial uncouplers that promote mitophagy reversed LPS/IFN- $\gamma$-mediated activation of macrophages and led to immunoparalysis with impaired bacterial clearance and lowered survival. In critically ill patients, we showed that mitophagy was inhibited in blood monocytes of patients with sepsis as compared with nonseptic patients. Overall, this work demonstrates that the inhibition of mitophagy is a physiological mechanism that contributes to the activation of myeloid cells and improves the outcome of sepsis.
\end{abstract}

\section{Introduction}

Sepsis is one of the leading causes of morbidity and mortality in intensive care units (ICUs) (1). Strategies that blunt inflammatory responses have failed to improve survival, whereas those that enhance host defenses have recently gained more attention (2, 3). Myeloid cells have a crucial role in the clearance of pathogens. After an initial inflammatory phase, classically activated macrophages (M1) undergo reprogramming to an alternate M2 phenotype (4). This second phase contributes to secondary immune suppression, known as immunoparalysis, which occurs during sepsis and counteracts the removal of infectious agents to ultimately worsen pathological conditions (5). The pivotal role of mitochondria in immune cells and immunity is now widely appreciated (68). In rodent models of sepsis, alterations of mitochondrial function have been described in the heart, the muscle, and the liver

Authorship note: DP and FM contributed equally to this work. FM is deceased. Conflict of interest: The authors have declared that no conflict of interest exists. Copyright: (5) 2020, American Society for Clinical Investigation.

Submitted: June 11, 2019; Accepted: July 29, 2020; Published: October 5, 2020.

Reference information: J Clin Invest. 2020;130(11):5858-5874.

https://doi.org/10.1172/JCl130996.
(9-11). Reduced activity of the mitochondrial respiratory chain has also been demonstrated in blood leukocytes of septic patients (12). These mitochondrial dysfunctions have been proposed to trigger the activation of the NLRP3 inflammasome in macrophages, leading to the release of IL-1 $\beta$, in particular through mitochondrial ROS (mROS) (13-15).

More broadly, alterations of mitochondria have a prominent role in multiorgan failure in septic patients (16). Mitochondrial quality is tightly linked to mitophagy and mitochondrial dynamics. Classically, defective mitochondria have decreased mitochondrial membrane potential $(\Delta \psi \mathrm{m})$ and enhanced fission. This leads to the stabilization and accumulation of PINK1 on the outer mitochondrial membrane (OMM), which activates the E3 ubiquitin-ligase Parkin and leads to its recruitment onto mitochondria. Parkin then decorates mitochondria with ubiquitin chains, which are sequentially phosphorylated by PINK1. The accumulation of phospho-ubiquitin chains on the OMM triggers the recruitment of the autophagy receptors and commits damaged mitochondria to mitophagy (17, 18). In rodent models of sepsis, prolonged impairment of mitophagy due to the ablation of Pink1 or Parkin genes results in reduced survival $(13,19)$. By contrast, adoptive transfer of bone marrow deficient in Dj-1, a PINK1 and Parkin partner $(20,21)$, protects the 
recipient mice against sepsis (22). Therefore, the role of mitophagy in immune cells and host defense remains to be clarified.

Mitochondrial function and mitophagy are linked to mROS production $(14,23)$. ROS signaling and oxidative stress have a pivotal role in septic conditions $(23,24)$. LPS-induced nitric oxide production, which inhibits complex I (CI), complex III (CIII), and complex IV (CIV) of the electron transport chain (ETC), is thought to promote mROS production (7). In activated macrophages, oxidation of succinate at complex II (CII) significantly augments mROS production. This results in the stabilization and activation HIF- $1 \alpha$ and the subsequent release of IL-1 $\beta$ (8). LPS-mediated increase in CII activity was also shown to contribute to pathogen clearance (25). Additionally, activation of TLR 1-2-4 signaling increases mROS production through TRAF6-dependent destabilization of CI. mROS was proposed to directly contribute to macrophage bactericidal activity $(26,27)$. Interestingly, the inhibition of mitophagy has been described as a major source of mROS (14). So far, investigations in mouse models deficient for mitophagy argue in favor of a deleterious role of the inhibition of mitophagy in the outcome of sepsis. Here, we investigated the role of mitophagy in macrophage activation through in vitro and in vivo approaches and assessed the consequences of its modulation in mouse models of sepsis and in critically ill patients.

\section{Results}

LPS enhances mitochondrial ROS production through the inhibition of mitophagy in macrophages. Enhanced production of mROS in macrophages has been proposed to result from the activation of TLR 1-2-4 signaling (26). We also noticed an increase in mROS with Pam2CSK4 (TLR2-6 agonist), Poly(I:C) (TLR3 agonist), and R848 (TLR7-8 agonist) (Figure 1A). The quantification of mitochondrial density with MitoTracker Green FM (a mitochondrial dye insensitive to $\Delta \psi_{\mathrm{m}}$ ) by flow cytometry revealed that increased mitochondrial density was a specific signature of the exposure to LPS (a component of gram-negative bacteria), relative to mROS production that was similarly induced by different TLR agonists (Figure 1A). An increase in mitochondrial density was also observed with live gram-negative bacteria (E. coli O55:B5), but not with the gram-positive bacterial strain B. subtilis (Supplemental Figure 1A; supplemental material available online with this article; https://doi.org/10.1172/JCI130996DS1). This raises the possibility that the activation of TLR4 signaling promoted mROS production through a mechanism distinct from other TLRs. The inhibition of mitophagy is a major source of mROS (14). Similar to LPS-treated raw 264.7 macrophages, raw 264.7 macrophages exposed to pharmacological inhibitors of mitophagy (mdivi-1, 3-methyladenine (3-MA), apigenin, and 4,5,6,7-tetrabromobenzotriazole; see Supplemental Methods) displayed a concomitant increase in mROS production and mitochondrial density (Figure $1 \mathrm{~B}$ and Supplemental Figure 1, B and C). This rise in mROS production and mitochondrial density induced by LPS was markedly enhanced by IFN- $\gamma$ cotreatment, and thus appeared as a feature of classically activated macrophages (Figure 1C). This early rise in mitochondrial density was also observed by flow cytometry and microscopy in activated raw 264.7 macrophages transfected with an expression vector coding for LSSmOrange-cytochrome C oxidase subunit VIII (MitoOrange) (Supplemental Figure 1D).
The fast time course of LPS- and LPS/IFN- $\gamma$-mediated increase in mitochondrial density in macrophages associated with enhanced mROS production (Figure 1D and Supplemental Figure 1A) suggests that this event stems from an inhibition of mitophagy rather than from mitochondrial biogenesis, which occurs more slowly (28). Hence, we measured the spectral shift of mitochondrial-targeted mKeima (mt-mKeima) by flow cytometry, which specifically monitors autophagosomal engulfment of mitochondria (see Supplemental Methods). Exposure of raw 264.7 macrophages to LPS/IFN- $\gamma$ for 6 hours or 18 hours resulted in an increased signal corresponding to the excitation at neutral $\mathrm{pH}$ (no mitophagy), whereas the signal from mt-mKeima at acidic $\mathrm{pH}$ (mitophagy) was reduced. Consequently, the $\mathrm{pH} 4$ to $\mathrm{pH} 7$ ratio of mt-mKeima was reduced upon LPS/IFN- $\gamma$ exposure (Figure $1 \mathrm{E}$, upper), indicating a marked reduction in mitophagy in activated macrophages. By contrast, as previously reported in the literature, quantification of autophagic vacuoles using CYTO-ID (Figure 1E, lower) confirmed that LPS/IFN- $\gamma$ treatment induced autophagy in a time-dependent manner. LPS-induced autophagy has been shown to play an important role in macrophage activation and phagocytosis (29). Our data suggested that although overall autophagy increased, organelle-targeted autophagy processes, such as mitophagy, did not follow a similar trend. To further confirm our observations, we also assessed the protein levels of several mitophagy (PINK1, Parkin, DJ-1) and mitochondrial fission (DRP-1) markers, which were all reduced in a time-dependent manner in bone marrow-derived macrophages (BMDMs) (Figure 1F), as well as in raw 264.7 macrophages (Supplemental Figure 1E). Reduced levels of Parkin, PINK1, and their partner DJ-1 (Park7) have been linked to defective mitophagy and autosomal recessive forms of Parkinson disease (30).

Inhibition of mitophagy is a feature of activated macrophages. Stat1-deficient macrophages failed to commit to classical activation as confirmed in vivo in LPS-injected Stat1-deficient mice (Supplemental Figure 2A) and in vitro in Stat1-deficient BMDMs and raw 264.7 macrophages (Supplemental Figure 2, B and C). Noticeably, CD64 and CD80 M1 polarization markers were unresponsive to LPS in Stat1-deficient BMDMs and in STAT1 siRNA-transfected raw 264.7 macrophages (Supplemental Figure 2, B and C). Along with this, LPS-mediated inhibition of mitophagy was absent in Stat1deficient BMDMs and raw 264.7 macrophages, as indicated by the normalization of the protein levels of mitophagy markers (Figure $1 \mathrm{~F}$ and Supplemental Figure 1E). These data highlight the inhibition of mitophagy as a feature of activated macrophages.

Mitochondrial uncouplers are classically used to promote mitophagy $(18,28)$. As expected, exposure of raw 264.7 macrophages to 2,4-dinitrophenol (2,4-DNP) or carbonyl cyanide m-chlorophenyl hydrazone (CCCP) promoted mitophagy and increased PINK1, DJ-1, and DRP1 protein levels (Figure $1 G$ and Supplemental Figure 1F). 2,4-DNP and CCCP treatment reversed LPS/IFN- $\gamma$-mediated inhibition of mitophagy, as indicated by the normalization of PINK1, Parkin, DJ-1, and DRP1 levels (Figure 1G and Supplemental Figure 1F). The recruitment of Parkin onto mitochondria is also a key determinant of the commitment of mitochondria to mitophagy. In most cell types, at steady state, PINK1 and Parkin are indiscernible in mitochondria. Here, in quiescent mouse peritoneal macrophages, we observed a colocalization of PINK1 and Tom20 (an OMM marker) on round-shaped mitochondria (Figure 2A). Parkin was also detected on isolated mitochondria in raw 264.7 mac- 
A

A
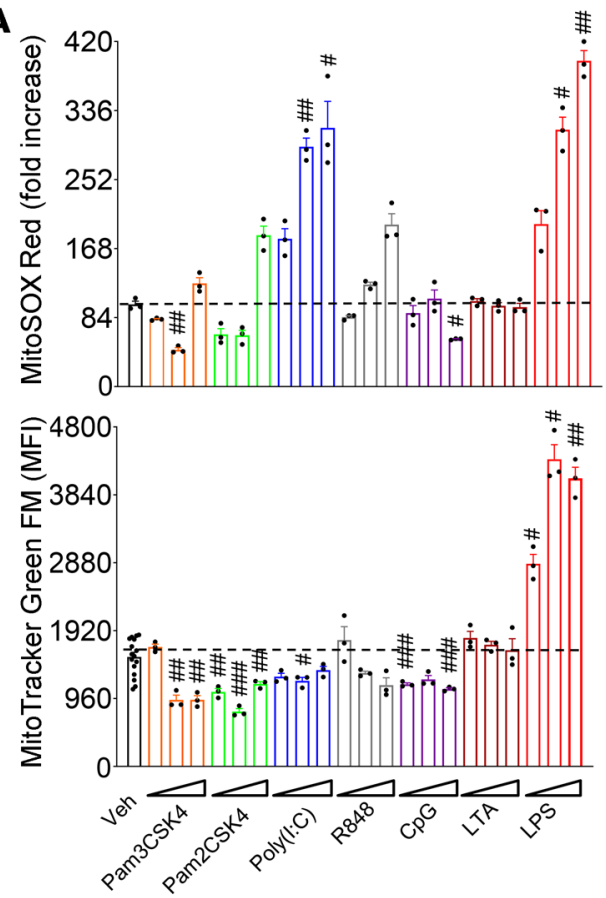

E
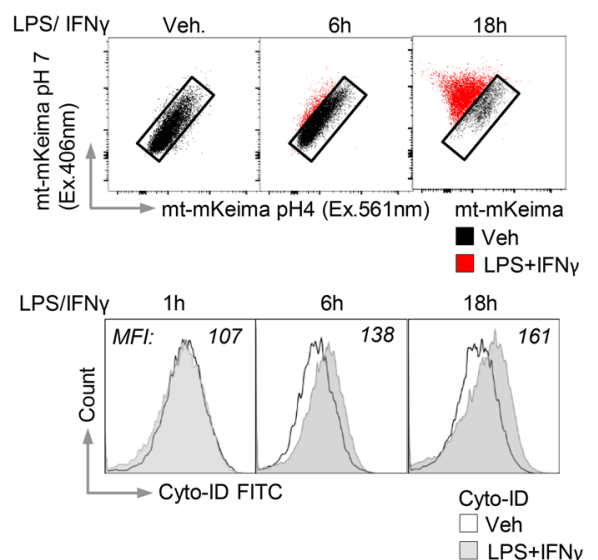

$\square$ LPS+IFN
B

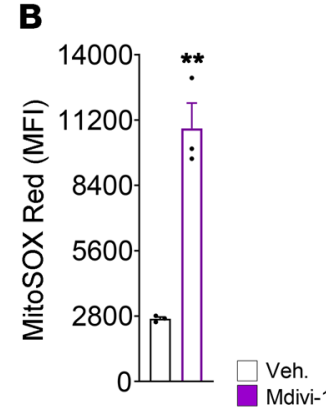

C
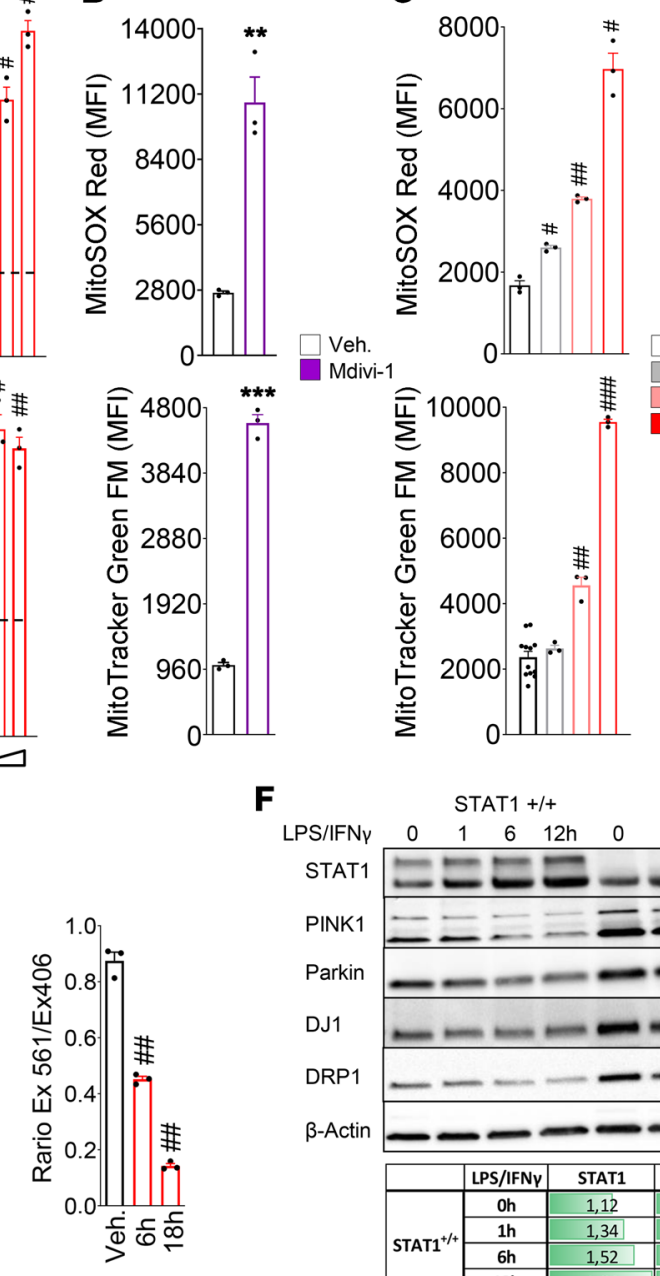

F

G
D

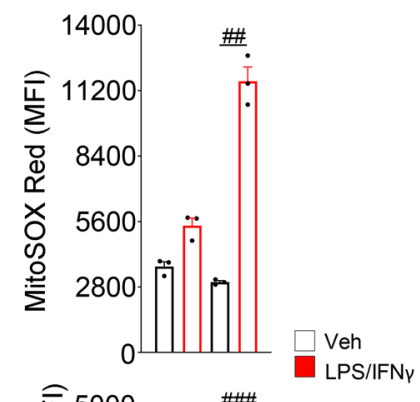

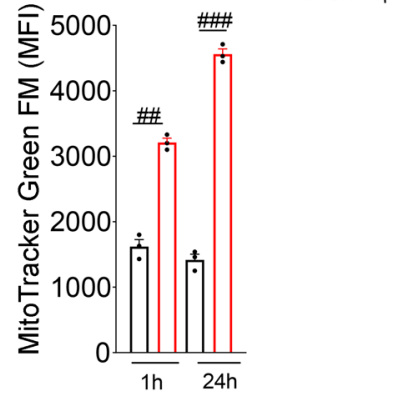
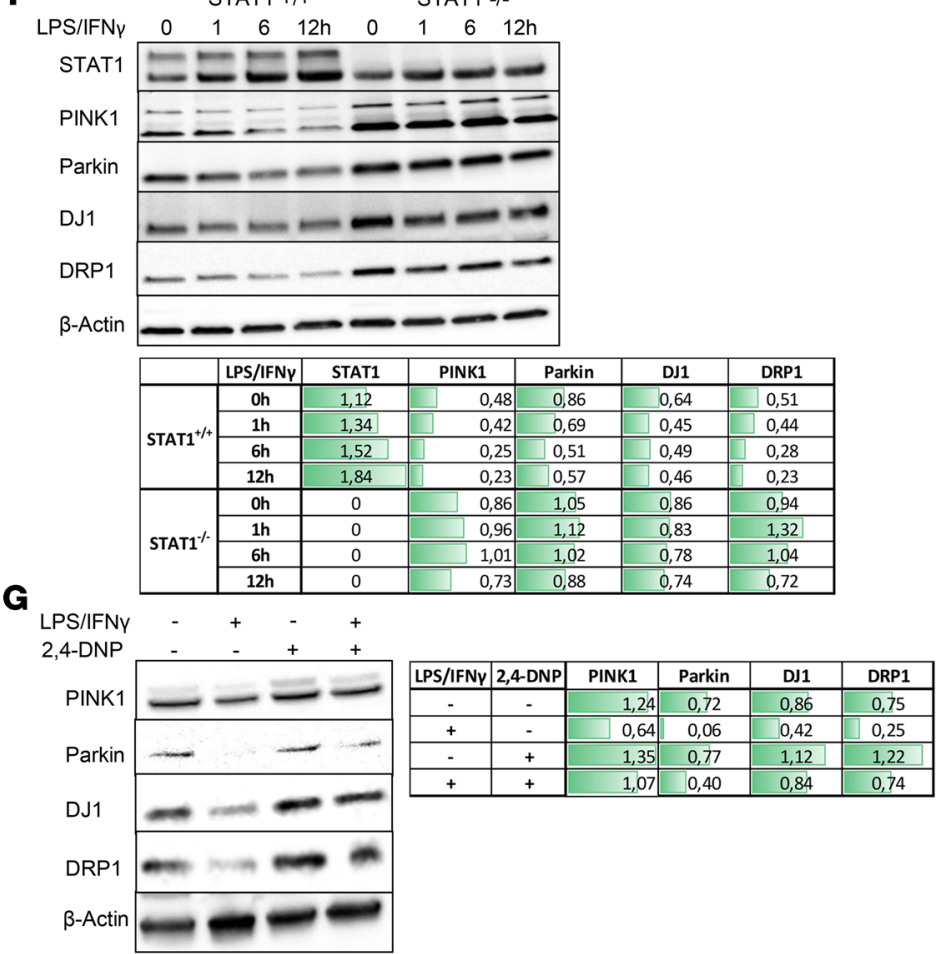

Figure 1. Macrophage activation is associated with the early inhibition of mitophagy. (A-D) Flow cytometry assessment of mitochondrial ROS (upper) and mitochondrial density (lower) in raw 264.7 macrophages (A) exposed to TLR agonists, (B) mitophagy inhibitor (mdivi-1), or (C and $\mathbf{D})$ to LPS and IFN- $\gamma$ alone or in combination for 24 hours or a specified duration ( $n=3$ per condition). (E) Flow cytometry assessment of mitophagy in raw 264.7 macrophages with mt-mkeima (upper) or autophagic vacuole formation with CYTO-ID autophagy detection kit (lower) in raw 264.7 macrophages exposed to LPS/IFN- $\gamma$ ( $n=3$ per condition). (F and G) Immunoblots of mitophagy and mitochondrial fission checkpoints on protein lysates from (F) stat $7^{+/+}$or stat $7^{-1-}$ BMDMs exposed to LPS/IFN- $\gamma$ or (C) from raw 264.7 macrophages exposed for 24 hours to LPS/IFN- $\gamma$ alone or in combination with a mitophagy inducer (2,4-DNP, $1 \mu \mathrm{M})$ (densitometry: ratio to $\beta$-actin is

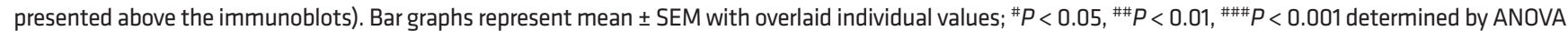
corrected for multiple comparisons; ${ }^{*} P<0.05$, ${ }^{* *} P<0.001$ determined by Student's $t$ test with Welch's correction.

rophages (Supplemental Figure 3A). This argues in favor of a high basal mitophagy flux in quiescent macrophages. The mitochondrial localization of PINK1 and the recruitment of Parkin onto mitochondria were, however, dampened upon LPS/IFN- $\gamma$-mediated macrophage activation (Figure 2A and Supplemental Figure 3A).
LPS/IFN- $\gamma$-dependent activation of macrophages is associated with high $\Delta \psi m$ and fused mitochondria. Mitophagy fluxes are intimately linked to alteration in mitochondrial dynamics and cell bioenergetics. Whereas the induction of mitophagy is associated with an increase in mitochondrial fission and a drop in $\Delta \psi \mathrm{m}$, 


\section{A Peritoneal $\mathrm{M} \varphi$}
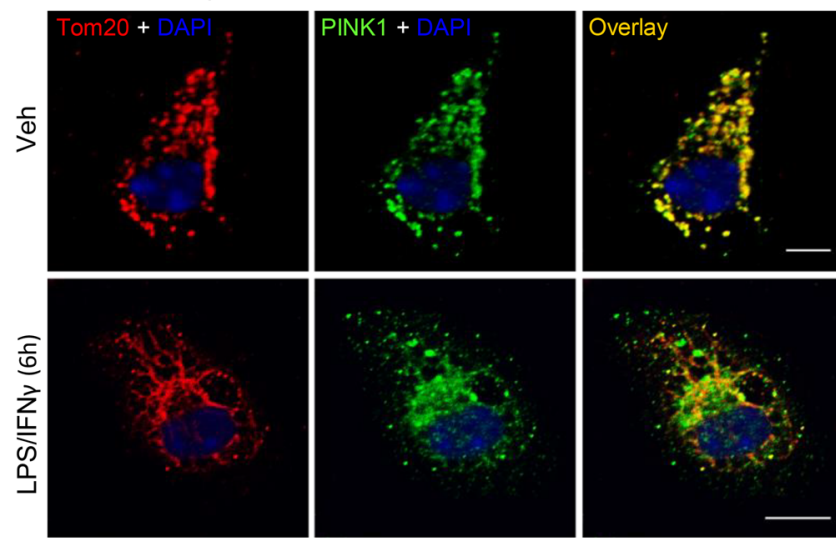

C
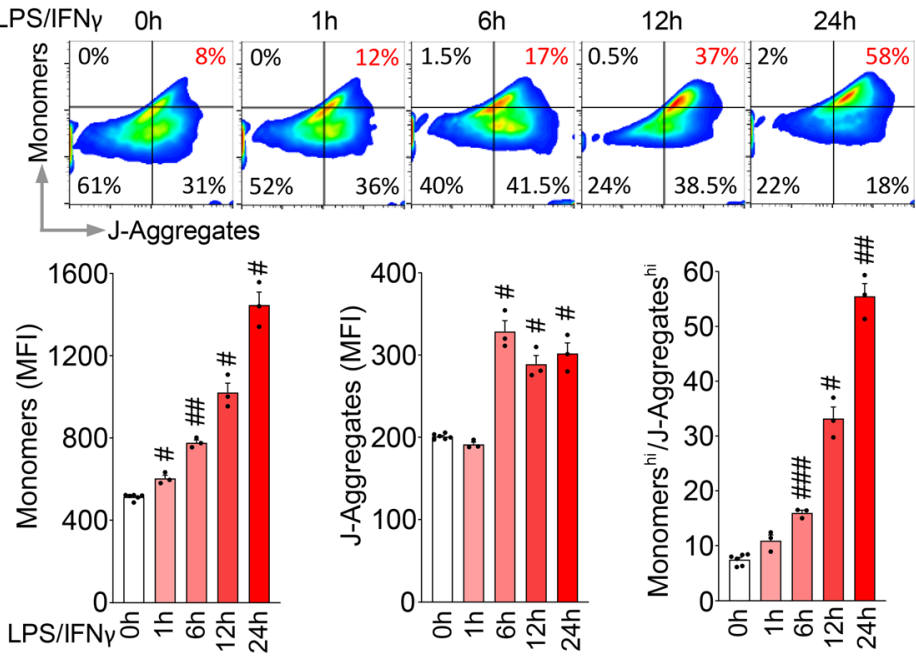

D
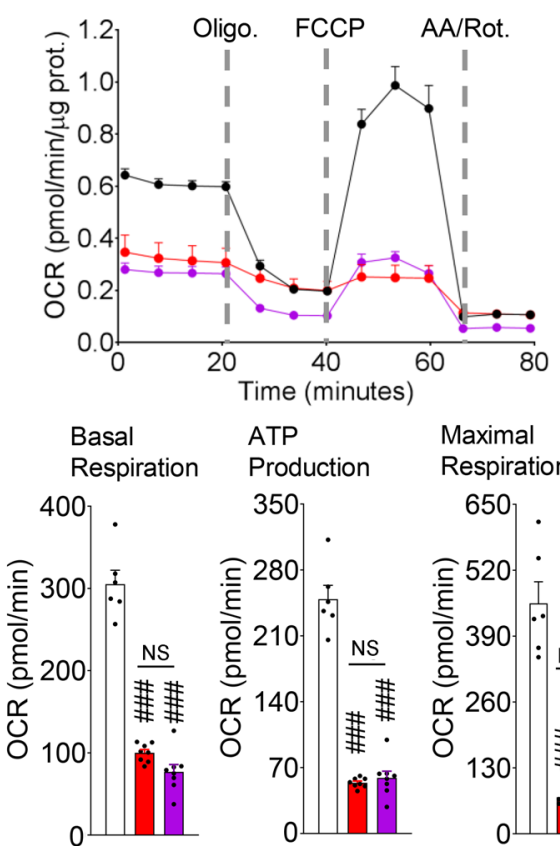

ATP Maximal

Production Respiration

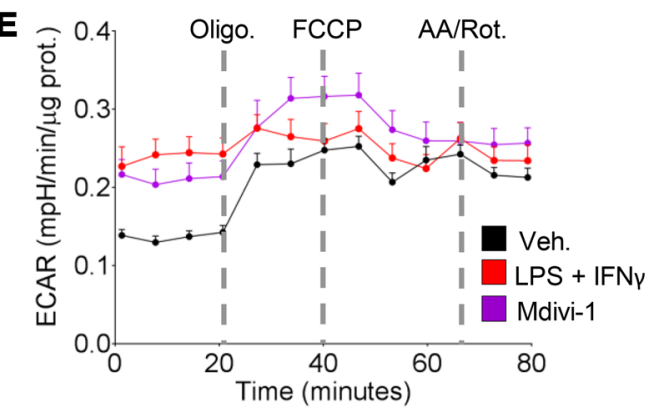

F
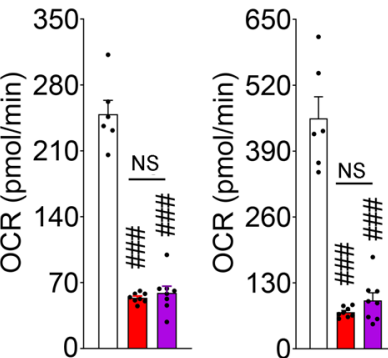

$\square$ Veh.

$\square \mathrm{LPS}+\mathrm{IFN}$

Mdivi-1
B Raw 264.7

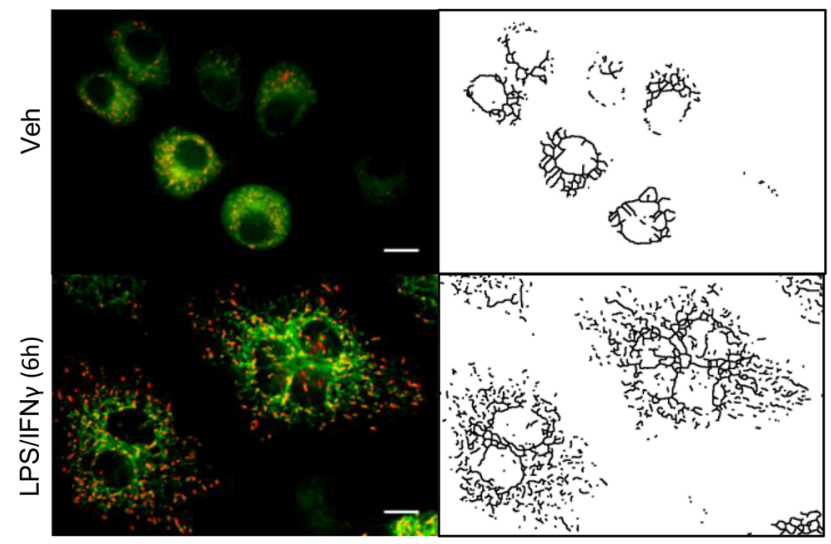

Figure 2. LPS/IFN- $\gamma$-mediated inhibition of mitophagy is associated with metabolic reprogramming of macrophages. (A and B) Mitochondrial network of (A) mouse peritoneal macrophages or (B) raw 264.7 macrophages exposed to vehicle or LPS/IFN- $\gamma$ for 6 hours (A) immunostained for Tom20 in combination or not with PINK1 or (B) stained with JC-1 (right panels represent the image binarization of JC-1 stained macrophages). Scale bars: $10 \mu \mathrm{m}$. (C) Flow cytometry assessment of mitochondrial density and $\Delta \psi \mathrm{m}$ in raw 264.7 macrophages exposed to LPS/IFN- $\gamma$ and stained with JC- $1(n=3$ per time point). ( $\mathbf{D}$ and $\mathbf{E}$ ) (D) Oxygen consumption (OCR) and extracellular acidification (ECAR) profile measured with Seahorse XFe96 analyzer on BMDMs exposed to vehicle, LPS/IFN- $\gamma$ for 6 hours, or mdivi- 1 for 24 hours ( $n=8$ per condition). (F) Flow cytometry assessment of $\Delta \psi \mathrm{m}$ with TMRM in raw 264.7 macrophages exposed to LPS/IFN- $\gamma$ for 6 hours ( $n=3$ per condition). (G) Cell death assessed by low cytometry (annexin V-PI) in raw 264.7 macrophages exposed to vehicle or LPS/IFN- $\gamma$ and 2,4 DNP alone or in combination for 18 hours. Bar graphs represent mean \pm SEM with overlaid individual values; ${ }^{\#} P<0.05,{ }^{\# \#} P<0.01$, $\# \# P<0.001$ determined by ANOVA corrected for multiple comparisons; ${ }^{* *} P<0.01,{ }^{* *} P<0.001$ determined by Student's $t$ test with Welch's correction. Veh, vehicle. 
A Blood Monocytes
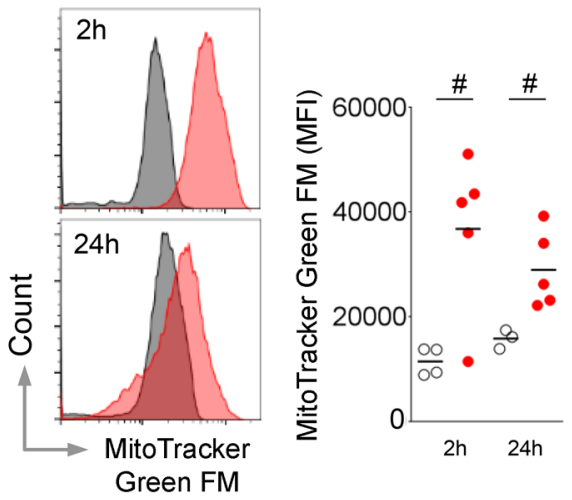

B Blood Inflammatory Monocytes

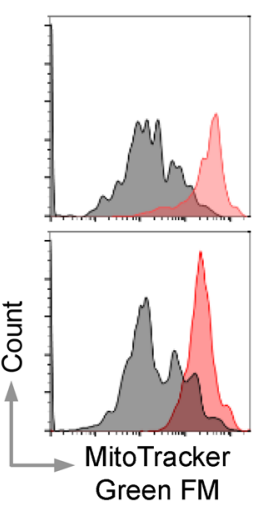

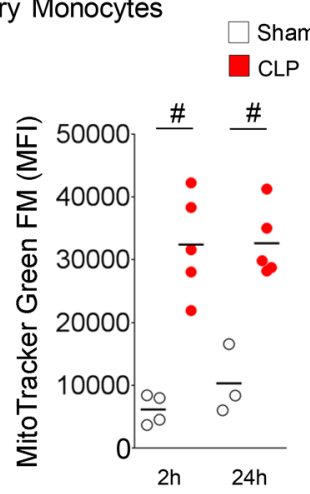

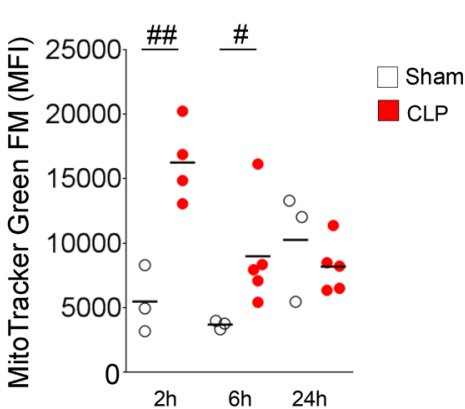

Figure 3. Polymicrobial infection triggers the early inhibition of mitophagy in myeloid cells. (A and B) Flow cytometry assessment of the mitochondrial density in (A) total monocytes (CD45 ${ }^{+}$CD115 $5^{+}$ CD11 $b^{\text {hil }}$ ) and in (B) inflammatory monocyte subpopulation (CD45 ${ }^{+}$CD115+ CD11b hi Ly6 ${ }^{\mathrm{hi}}$ ) in the blood of (57BL6/J mice after sham or CLP surgery ( $n=3-4$ sham; $n=5$ CLP). (C) Flow cytometry assessment of the mitochondrial density of peritoneal macrophages (CD45 $\mathrm{F} 4 / 80^{\text {hi }} \mathrm{CD} 11 \mathrm{~b}^{\mathrm{hi}}$ ) in peritoneal fluid of mice treated as in $\mathbf{A}$ and $\mathbf{B}$. (D) Flow cytometry assessment of mitochondrial density in CD64 ${ }^{\text {hi }}$ Mitotrackeri subpopulation of peritoneal macrophages of mice treated as in $\mathbf{A}$ and $\mathbf{B}$. Graphs with plots represent mean plus individual values; ${ }^{\#} P<0.05,{ }^{\#} P<$ $0.01,{ }^{\# \#} P<0.001$ determined by ANOVA corrected for multiple comparisons.

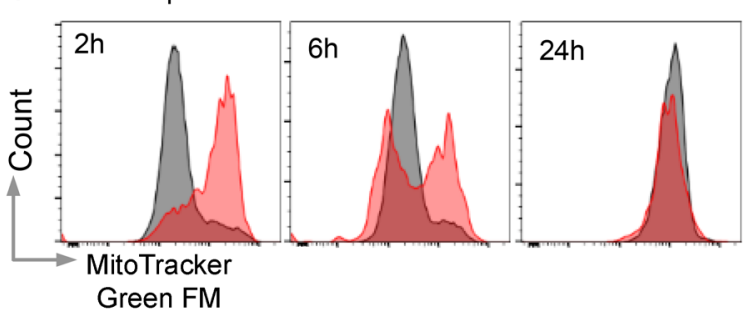

D CD64 ${ }^{\text {hi }}$ MitoTracker ${ }^{\text {hi }} M \varphi$

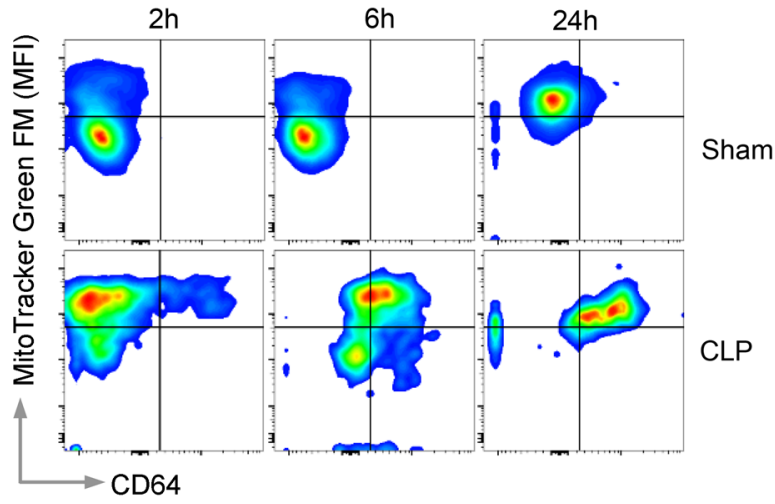

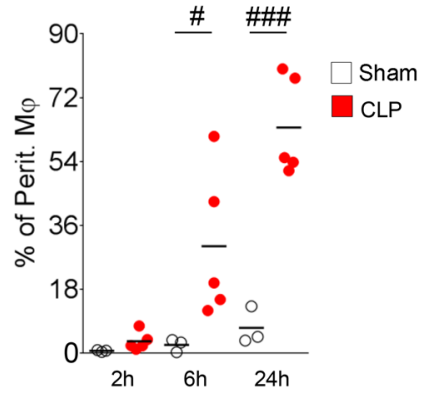

enhancement of mitochondrial fusion and high $\Delta \psi \mathrm{m}$ are observed concomitantly with the inhibition of mitophagy (31). In line with this, raw 264.7 macrophages and peritoneal macrophages treated with LPS/IFN- $\gamma$ for 6 hours displayed a marked reconfiguration of the mitochondrial network, which became fused, denser, and more connected (Figure 2A and Supplemental Figure 3B). JC-1 dye allowed visualization of mitochondria with both low (JC-1 monomers, green) and high $\Delta \psi \mathrm{m}$ (JC-1 aggregates, red) (Figure 2B). Image binarization of JC-1-stained macrophages confirmed the robust increase in the mitochondrial network in LPS/IFN- $\gamma$-treated macrophages as early as 6 hours after treatment (Figure 2B).

Whereas mainly low- $\Delta \psi \mathrm{m}$ mitochondria were observed in quiescent macrophages, the proportion of high- $\Delta \psi \mathrm{m}$ mitochondria (JC-1 aggregates) rapidly increased after exposure to LPS/IFN- $\gamma$ (Figure 2B). The time-dependent increase in $\Delta \psi \mathrm{m}$ upon macrophage activation was confirmed by flow cytometry in raw 264.7 macrophages (Figure 2C). Interestingly, JC-1 monomers were also markedly increased, further confirming that LPS/IFN- $\gamma$ increased the mitochondrial density in macrophages; and this occurred with a strong representation of high- $\Delta \psi \mathrm{m}$ mitochondria, as shown by the concomitant increase in JC-1 aggregates (Figure $2 \mathrm{C}$ ). The enhancement of $\Delta \psi \mathrm{m}$ in activated macrophages was confirmed by TMRM staining (Figure 2F).

Inhibition of mitophagy contributes to the survival of activated macrophages. Within the same timeframe, the oxygen consumption rate (OCR) was reduced in BMDMs exposed to LPS/IFN- $\gamma$ for 6 hours, whereas the extracellular acidification rate (ECAR) was enhanced (Figure 2, D and E). This specificity of LPS-mediated metabolic rewiring in myeloid cells compared with other TLR agonists was also reported by others (32). This metabolic feature that was maintained 24 hours after exposure to LPS/IFN- $\gamma$ (data not shown) underlies the metabolic rewiring of LPS-activated macrophages toward glycolysis $(6,7)$. It is noteworthy that the OCR and ECAR profiles of BMDMs exposed to the mitophagy inhibitor mdivi-1 were similar to those of classically activated macrophages (Figure 2, D and E). Despite the strong reduction of OCR 


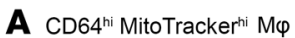
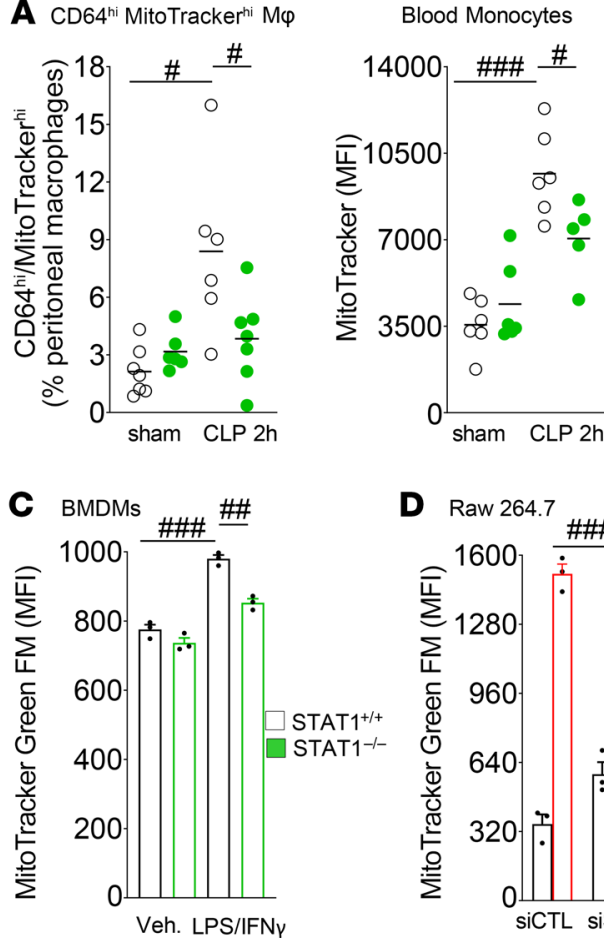

B

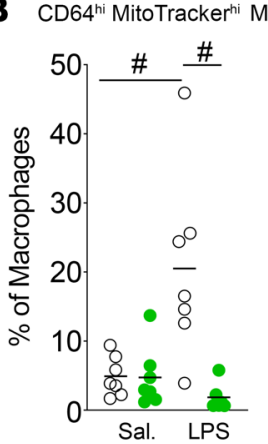

D Raw 264.7

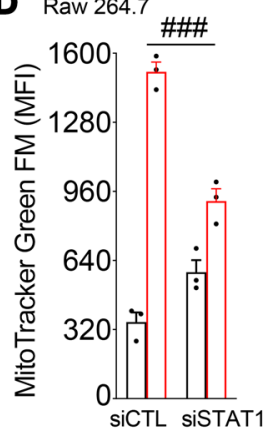

E Raw 264.7

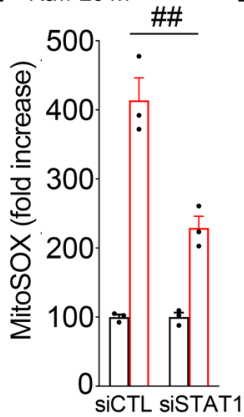

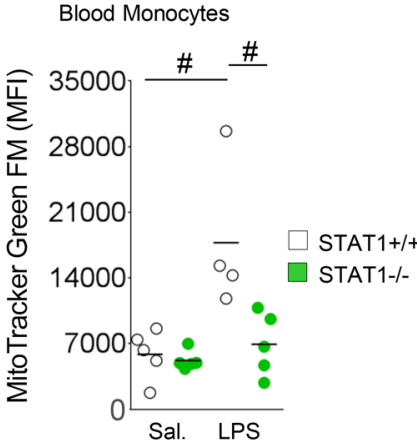

F $\quad$ Raw 264.7

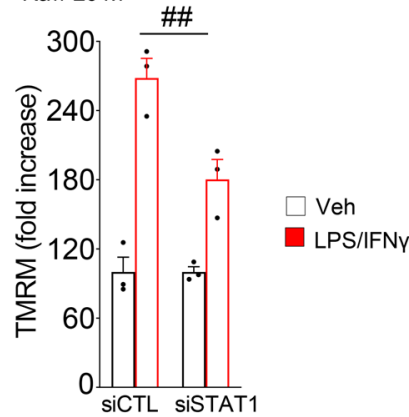

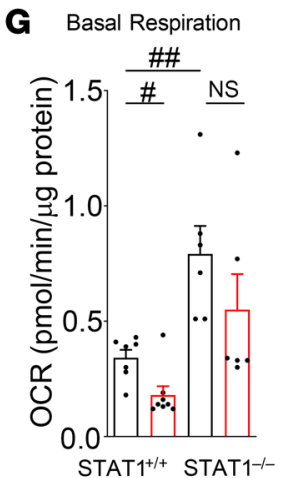
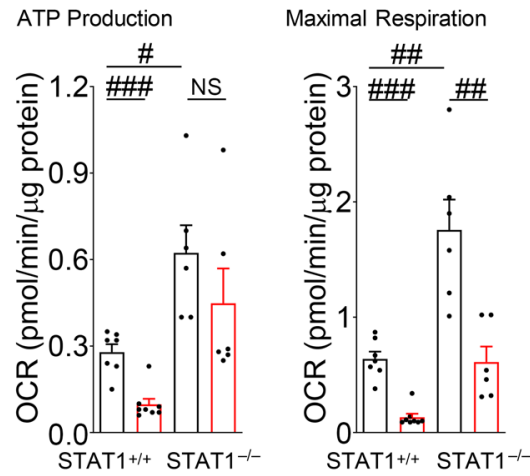

Figure 4. Polymicrobial infection and endotoxemia trigger the early inhibition of mitophagy in myeloid cells in a STAT1-dependent manner. (A and B) Flow cytometry assessment of CD64 ${ }^{\text {hi }}$ Mitotracker hi peritoneal macrophage subpopulation and mitochondrial density in blood monocytes of stat $7^{+/+}$or stat1 ${ }^{-1-}$ mice after (A) sham or CLP surgery ( $n=6-7$ sham; $n=5-7$ CLP) or (B) i.p. injection of saline (sal.) or LPS (0.5 mg $/ \mathrm{kg}, 24 \mathrm{hours)}(n=5-7 \mathrm{sal} . ; n=5-7$ LPS). (C and D) Flow cytometry assessment of mitochondrial density in (C) stat $1^{+/+}$or stat ${ }^{-1-}$ BMDMs or in (D) raw 264.7 macrophages targeted with control (CTL) or stat1 siRNA and then exposed to vehicle or LPS/IFN- $\gamma$ for 24 hours. (E and F) Flow cytometry assessment of (E) mitochondrial ROS production and (F) $\Delta \psi \mathrm{m}$ in raw 264.7 macrophages targeted with CTL or stat1 siRNA and then exposed to vehicle or LPS/IFN- $\gamma$ for 24 hours. (C) Oxygen consumption profile measured with Seahorse XFe96 analyzer on stat1 ${ }^{+/+}$or stat1 ${ }^{-1-}$ BMDMs exposed to vehicle or LPS/IFN- $\gamma$ for 6 hours ( $n=6-8$ per condition). Graphs with plots represent mean plus individual values; bar graphs represent mean \pm SEM with overlaid individual values; ${ }^{\#} P<0.05,{ }^{\# \#} P<0.01,{ }^{\# \# \# P<0.001}$ determined by ANOVA corrected for multiple comparisons. Veh, vehicle.

and the increase in ECAR, exposure to LPS/IFN- $\gamma$ did not increase cell death (Figure $2 \mathrm{G}$ ). The survival of LPS/IFN- $\gamma$-exposed macrophages could be explained by mitochondrial fusion $(33,34)$. By contrast, the stimulation of mitophagy with 2,4-DNP in activated raw 264.7 macrophages enhanced cell death. Therefore, inhibition of mitophagy may also contribute to cell survival in conditions of low respiration.

LPS triggers the inhibition of mitophagy in myeloid cells in vivo. Gram-negative bacteria are the predominant species in the cecal ligation and puncture (CLP) model of polymicrobial infection (35), which we used to explore the translational relevance of our in vitro findings. Female C57BL6/J mice underwent CLP or sham surgery. Mitophagy was assessed extemporaneously on freshly collected blood peritoneal leukocytes through the measurement of mitochondrial density by flow cytometry. Samples were collected after CLP or sham surgery at indicated time points (Figure 3 and Supplemental Figure 4). Blood and peritoneal leukocytes were incubated for 10 minutes with MitoTracker Green FM before immunostaining and then processed for flow cytometry analysis. In the blood, the proportion of monocytes $\left(\mathrm{CD} 45^{+} \mathrm{CD} 115^{+}\right)$and the inflammatory monocyte subpopulation $\left(\mathrm{CD} 45^{+} \mathrm{CD} 115^{+} \mathrm{CD} 11 \mathrm{~b}^{\mathrm{hi}}\right.$ Ly $6 C^{\text {hi }}$ ) were not significantly affected by polymicrobial infection, except 24 hours after CLP for inflammatory monocytes (Supplemental Figure 4, C and D). By contrast, monocytes and the sub- 

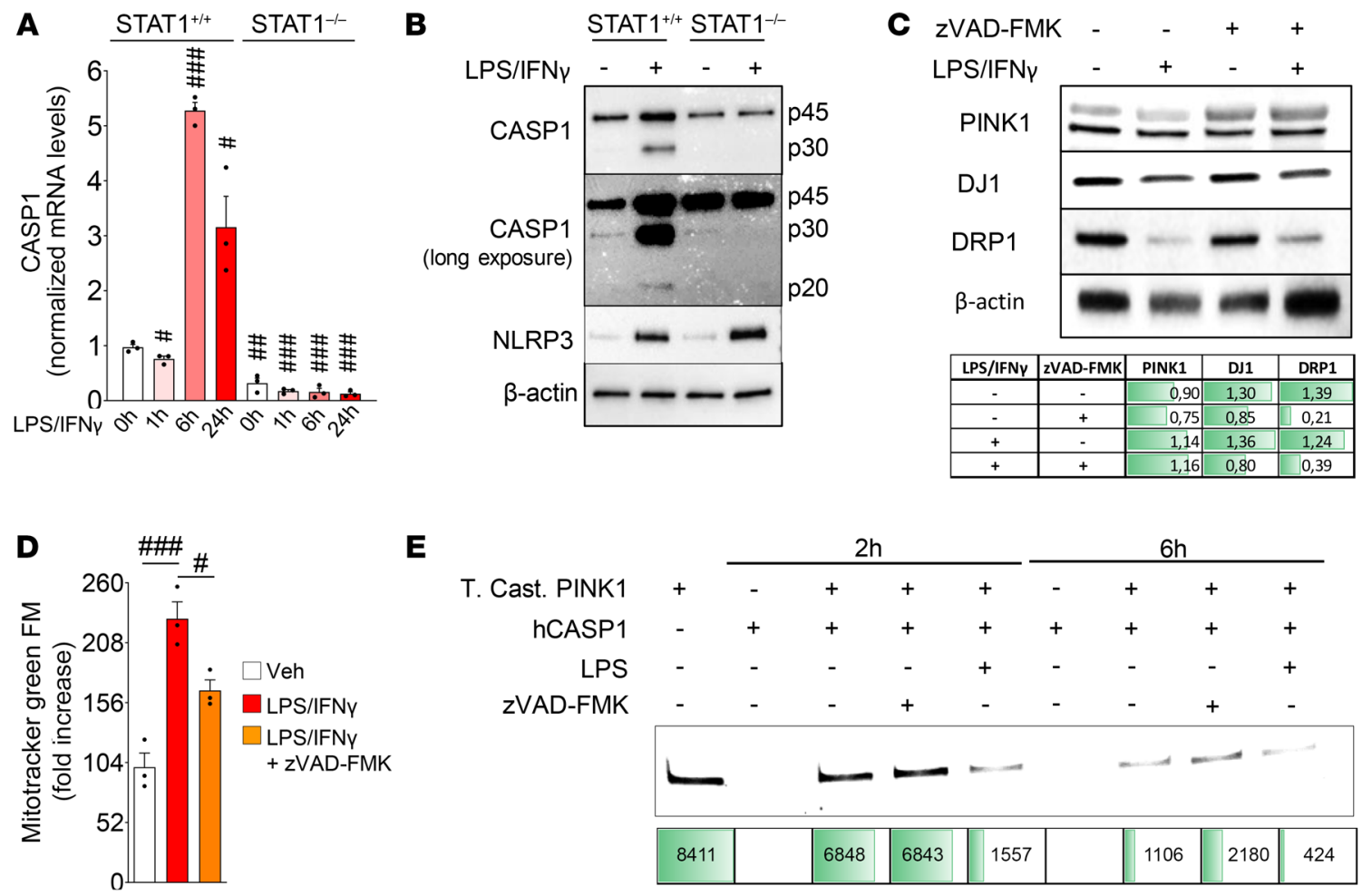

Figure 5. LPS/IFN- $\gamma$ inhibits mitophagy in macrophages through the STAT1-dependent regulation of caspase-1. (A and B) Caspase- 1 expression in stat $7^{+/+}$ or stat1 ${ }^{-1-}$ BMDMs exposed to vehicle or LPS/IFN- $\gamma$ for 18 hours or indicated duration assessed by (A) qPCR or (B) immunoblotting ( $n=3$ per condition). (C and D) Assessment by (C) immunoblotting or (D) flow cytometry of the impact of zVAD-FMK-dependent inhibition of caspases on LPS/IFN- $\gamma$-dependent inhibition of mitophagy in raw 264.7 macrophages exposed to vehicle or LPS/IFN- $\gamma$ for 18 hours ( $n=3$ per condition) (densitometry: ratio to $\beta$-actin is presented above the immunoblots). (E) Proteolytic activity of recombinant human caspase-1 (hCASP1) against recombinant Tribolium castaneum PINK1 (T. cast. PINK1) in the presence of ZVAD-FMK and LPS $(100 \mathrm{ng} / \mathrm{mL})$ alone or in combination. Protein levels were assessed with 2,2,2-TCE gels after UV transillumination (the table below $\mathbf{C}$ presents densitometry data). Bar graphs represent mean \pm SEM with overlaid individual values; ${ }^{\#} P<0.05,{ }^{\#} P<0.01, \# \# \#<$ 0.001 determined by ANOVA corrected for multiple comparison.

population of inflammatory monocytes showed a marked increase in mitochondrial density at 2 hours and 24 hours after infection (Figure 3, A and B). We also observed a robust increase in mitochondrial density in peritoneal macrophages at early time points ( 2 hours, 6 hours) after CLP surgery compared with sham surgery (Figure 3C). These changes were independent from the proportion of peritoneal macrophages (Supplemental Figure 4E).

The CD64 ${ }^{h i}$ Mitotracker ${ }^{h i}$ macrophage subpopulation is highly increased during sepsis. As expected, peritoneal macrophages from infected mice displayed a significant rise in the levels of the macrophage activation markers CD64 (Fc $\gamma \mathrm{RI}$ ) and CD80 (Supplemental Figure 4, F and G). The CD64 level in myeloid cells has been positively correlated with the severity of sepsis (36). Accordingly, the increase in the CD64 level in the peritoneal macrophages of mice receiving CLP was more pronounced compared with the CD80 level (Supplemental Figure 4G). We identified a subpopulation of $\mathrm{CD} 64^{\mathrm{hi}}$ macrophages with a high mitochondrial density (CD64 ${ }^{\text {hi }}$ Mitotracker ${ }^{\text {hi }}$ ) whose proportion in macrophages increased in a time-dependent manner In mice receiving CLP (Figure 3D). In noninfected animals, although the proportion of peritoneal CD $64^{\text {hi }}$ macrophages was extremely low, mitochondrial density was significantly higher than in $\mathrm{CD} 64^{\text {lo }}$ or $\mathrm{CD} 80^{\text {lo }}$ and $\mathrm{CD}^{\mathrm{hi}} \mathrm{h}^{\mathrm{hi}}$ macrophages (Supplemental Figure $4, \mathrm{H}$ and I). To con- firm that the phenotype of monocytes and macrophages observed after CLP surgery was due to LPS produced by gram-negative bacteria, we assessed the impact of low $(0.5 \mathrm{mg} / \mathrm{kg})$ and high $(15 \mathrm{mg} / \mathrm{kg})$ doses of i.p. injected LPS. Both doses led to a similar phenotype with an increase in mitochondrial density in peritoneal macrophages and blood monocytes, as well as a rise in the CD64 ${ }^{\text {hi }}$ Mitotrackerhi macrophage subpopulation as early as 1 hour after exposure (Supplemental Figure 4, J-L).

LPS mediates the inhibition of mitophagy in a STAT1-dependent manner. Stat1-deficient mice were defective with regards to the activation of macrophages (Supplemental Figure 2, A-C). This phenotype was associated with an absence of reduction of the protein levels of mitophagy and mitochondrial fission checkpoints (Figure $1 \mathrm{~F}$ and Supplemental Figure 1E). We further explored the impact of STAT1 invalidation on mitophagy and showed that Stat1-deficient mice also failed to show a CLP- and LPS-mediated increase in mitochondrial density at 2 hours in $\mathrm{CD} 64^{\text {hi }}$ peritoneal macrophages and blood monocytes (Figure 4, A and B). These observations were confirmed in vitro in BMDMs and raw 264.7 macrophages (Figure 4, C and D). Additionally, changes in mROS production, $\Delta \psi \mathrm{m}$, and respiration associated with LPS/IFN- $\gamma$ mediated inhibition of mitophagy were reduced or normalized in Stat1-deficient BMDMs (Figure 4, E-G). 
A

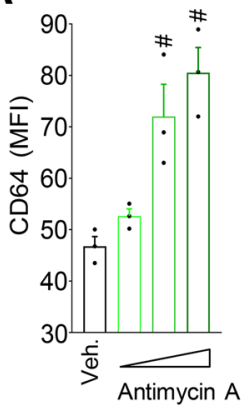

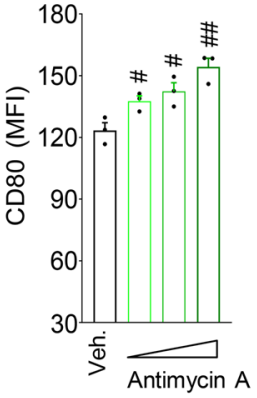

B

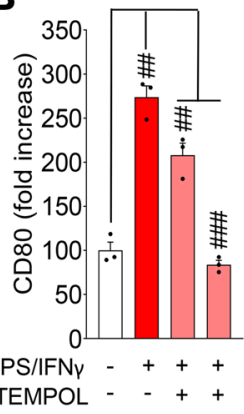

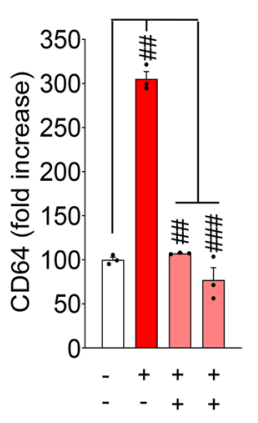

C

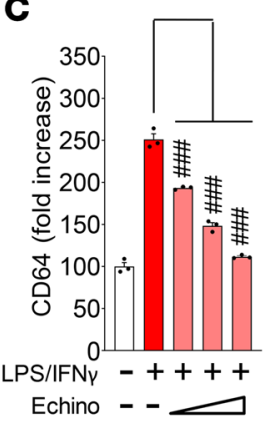

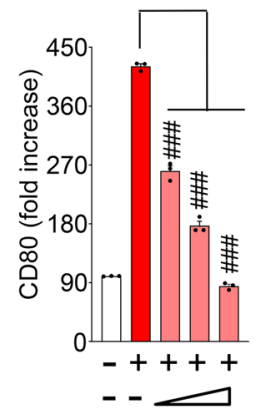

$\mathbf{F}$

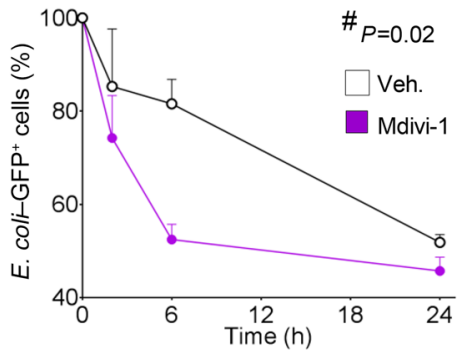

I

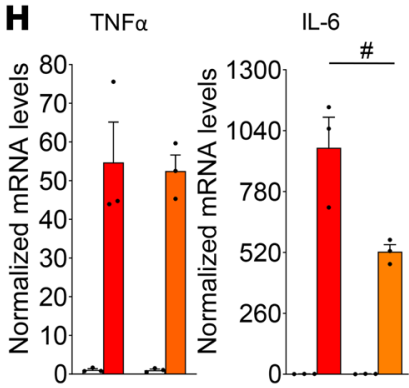

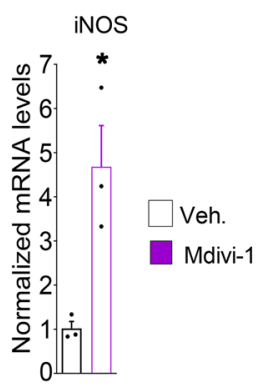

iNOS

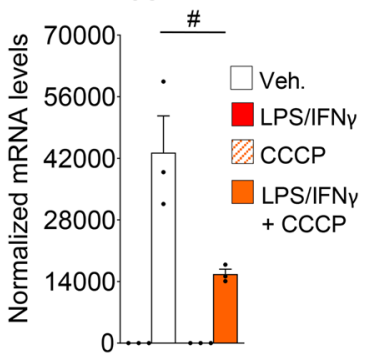

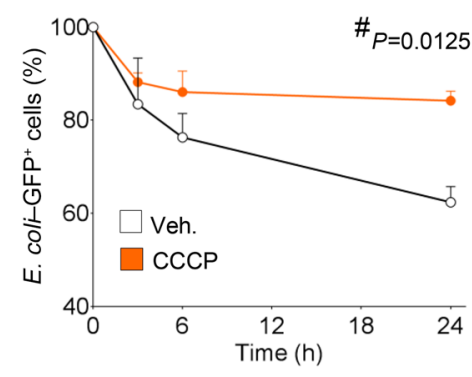

Figure 6. The inhibition of mitophagy triggers classical activation of macrophages through mitochondrial ROS. (A) Flow cytometry assessment of classical macrophage activation in raw 264.7 cells exposed to antimycin A for 6 hours. (B and $\mathbf{C}$ ) Flow cytometry assessment of classical macrophage activation in raw 264.7 cells exposed to LPS/IFN- $\gamma$ for 18 hours alone or in combination with (B) the mitochondrial ROS scavenger MitoTEMPOL or with (C) the HIF-1 $\alpha$ inhibitor echinomycin ( $n=3$ per condition). (D-I) Assessment of macrophage activation profile by ( $\mathbf{D}$ and $\mathbf{G})$ flow cytometry, (E and $\mathbf{H})$ gene expression, and (F and I) bactericidal activity in raw 264.7 cells (D, E, G, and $\mathbf{H}$ ) incubated (24 hours) or (F and I) preincubated (24 hours) with (D-F) the mitophagy inhibitor mdivi-1 or (G-I) the mitophagy inducer CCCP ( $n=3$ per condition). Bar graphs represent mean \pm SEM with overlaid individual values; ${ }^{\#} P<0.05$, ${ }^{\# \#} P<0.01$,

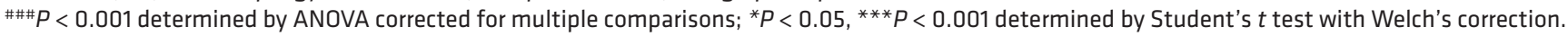

STAT1 inhibits mitophagy through inflammatory caspases 1 and 11. We then explored whether the above phenomenon was related to the inability of Stat1-deficient macrophages to commit to classical activation or to a STAT1-dependent modulation of mitophagy. Caspase-1 was shown to inhibit mitophagy by cleaving and inactivating Parkin subsequent to its translocation to mitochondria $(37,38)$. Interestingly, IFN- $\gamma$ was shown to regulate caspase-1 levels and activity (39). We showed here that STAT1-mediated LPS/IFN- $\gamma$-dependent regulation of caspase-1 at mRNA and protein levels in BMDMs did not affect NLRP3 levels (Figure 5, A and B). A similar pattern of regulation was observed for caspase-11 in raw 264.7 macrophages (Supplemental Figure 5, A and B), another key inflammatory caspase regulated and activated by LPS, which controls IL- $1 \beta$ secretion by potentiating caspase-1 activation (40). STAT1-dependent regulation of caspase-11 was only assessed in raw 264.7 macrophages because Stat1-deficient mice are on a 129SV genetic background and do not express caspase-11 (41). Similar to caspase-1 (37), the exposure of raw 264.7 macrophages to LPS/IFN- $\gamma$ led to the mitochondrial translocation of caspase-11 (Supplemental Figure 5C). The inhibition of caspases with zVAD-FMK (Figure 5C), as well as RNAi-mediated gene silencing of caspase-1 or caspase-11, resulted in the abrogation of an LPS/IFN- $\gamma$-mediated drop in PINK1 protein levels in raw 264.7 macrophages (Supplemental Figure 5D). This translated into a less-pronounced increase in mitochondrial density in activated macrophages exposed to zVAD-FMK or caspase-1 or -11 siRNA (Figure 5D and Supplemental Figure 5E). Surprisingly, LPS/IFN- $\gamma$-mediated Parkin downregulation was more pronounced upon caspase-1 or caspase-11 gene silencing (Supplemental Figure 5D). This observation suggests that PINK1 plays a central role in LPS/IFN- $\gamma$ mediated inhibition of mitophagy in macrophages. 
A

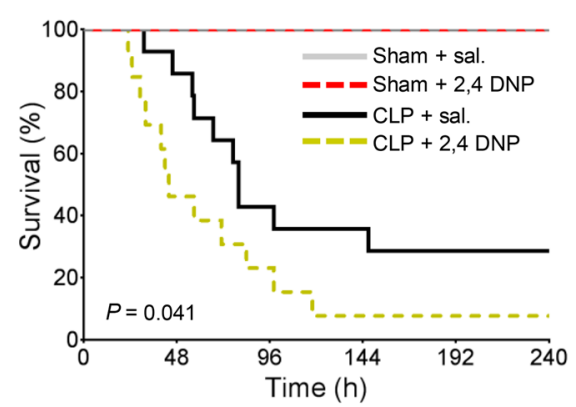

E

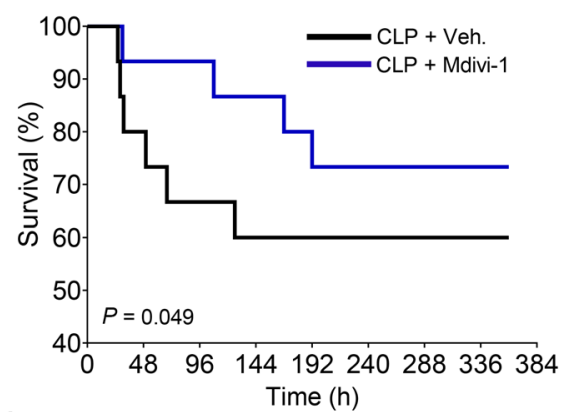

H

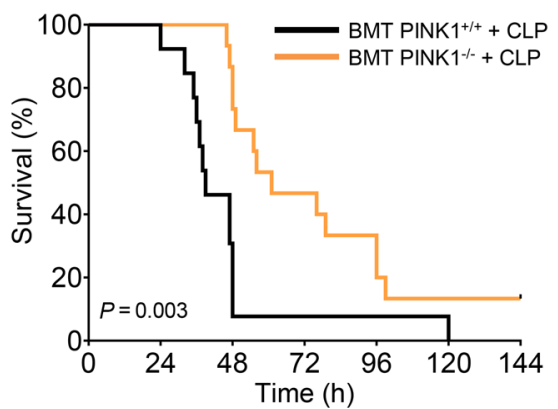

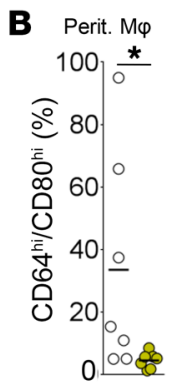

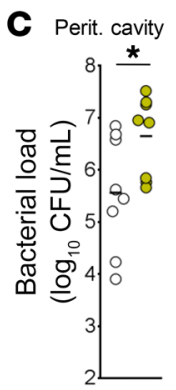

D

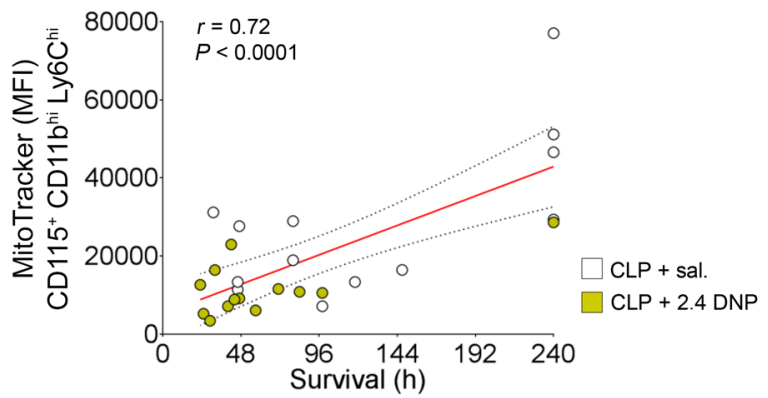

G Perit. cavity

F Perit. $M \varphi$
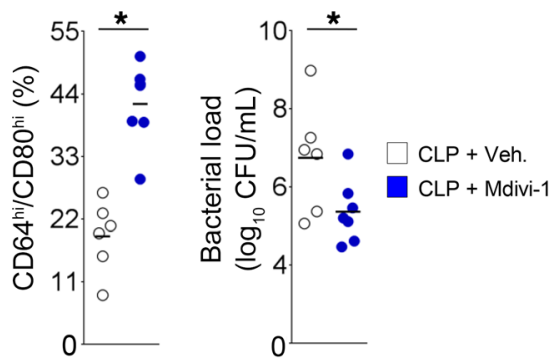

I Perit. $M \varphi$

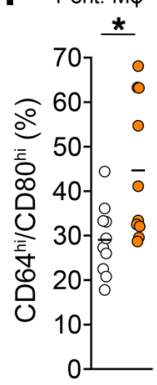

J Perit. cavity

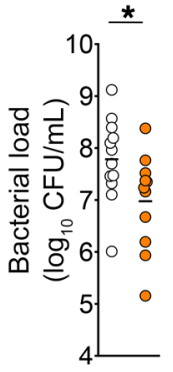

$\square$ BMT PINK1 $1^{+/+}+$CLP

$\square$ BMT PINK1 $1-$ CLP

Figure 7. Inhibition of mitophagy in myeloid cells protects against bacterial infection and improves survival during sepsis. (A) Survival curve of C57BL6/] mice treated with saline (sal.) or 2,4-DNP (10 mg/kg) 24 hours before sham or CLP surgery (sham, $n=5$ per group; CLP, $n=13$ per group). $P$ value was determined by Gehan-Breslow-Wilcoxon test. (B) Percentage of classically activated macrophages (percentage of CD111 ${ }^{\text {hi }}$ F4/80 ${ }^{\text {hi }}$ macrophages) in C57BL6/J mice treated as in $\mathbf{A}$ ( $n=7$ per condition). (C) Bacterial load in the peritoneal cavity of C57BL6/J mice treated as in $\mathbf{A}$ ( $n=8$ per condition). (D) Correlation of the mitochondrial density in Ly6C ${ }^{\text {hi }}$ blood monocytes 2 hours after CLP surgery versus the survival (in hours) after CLP surgery ( $\left.n=26\right)$. $P$ and $r$ values were determined by Spearman's rank correlation. (E) Survival curve of [57BL6/] mice treated with vehicle or mdivi-1 for 24 hours before CLP surgery (CLP + vehicle, $n=15$; CLP + mdivi-1, $n=15)$. $P$ value was determined by Gehan-Breslow-Wilcoxon test. (F and $\mathbf{G})$ Percentage of classically activated macrophages (F) (percentage of CD11 $\mathrm{b}^{\text {hi }} \mathrm{F} 4 / 80^{\text {hi }}$ macrophages) and bacterial load (C) in the peritoneal cavity of C57BL6/J mice treated as in $\mathbf{E}(n=6-7$ per condition) (H) Survival curve of C57BL6/J mice transplanted with Pink $7^{+/+}$(BMT Pink $1^{1^{++}}$) or Pink $1^{-/-}$bone marrow (BMT Pink $1^{-1-}$ ) 5 weeks before CLP surgery (BMT Pink $1^{+/+}$, $n=13$; BMT Pink $1^{-1-}, n=15$ ). $P$ value was determined by Gehan-Breslow-Wilcoxon test. (I) Flow cytometry assessment of the percentage of classically activated macrophages (percentage of $\mathrm{CD} 11 \mathrm{~b}^{\mathrm{hi}} \mathrm{F} 4 / 80^{\mathrm{hi}}$ macrophages) in the peritoneal cavity of mice that underwent transplantation and surgery as in $\mathbf{H}(n=11-12$ per group). (J) Bacterial load in the cavity of mice that underwent transplantation and surgery as in $\mathbf{H}$ ( $n=11-12$ per group). Graphs with plots represent mean plus individual values; ${ }^{*} P<0.05$ determined by Student's $t$ test with Welch's correction.

The inflammatory caspases 1 and 11 degrade PINK1. Caspase-11 was shown to degrade TRPC1 (42); caspase-1 is endowed with similar proteolytic properties. Therefore, we speculated that caspase- 1 and -11 could affect mitophagy by degrading PINK1. PINK1 has a pivotal role in mitochondrial quality control and is evolutionarily conserved (43). Using Cascleavage 2.0, Prosper 2.0, and CasDB, several conserved caspase cleavage sequences were identified in human, mouse, and Tribolium castaneum PINK1 (T. cast. PINK1). Recombinant Tribolium castaneum PINK1 that retains its kinase activity compared with human and mouse PINK1 (43-45) was used for further in vitro experiments. This active recombinant PINK1 was incubated with active recombinant human caspase-1 or -4 (the human orthologue of caspase-11) in the presence or absence of zVAD-FMK or LPS. Protein levels were assessed using polyacrylamide gels containing 2,2,2-trichloroethanol (TCE), which allowed fluorescent detection of proteins after UV exposure. Both recombinant caspase- 1 and $-4 / 11$ led to reduced PINK1 levels (Figure 5E and Supplemental Figure 5F). In agreement with the LPS-binding capacity of caspase-4/11 $(46,47)$, the reduction of PINK1 
$\mathbf{A}$

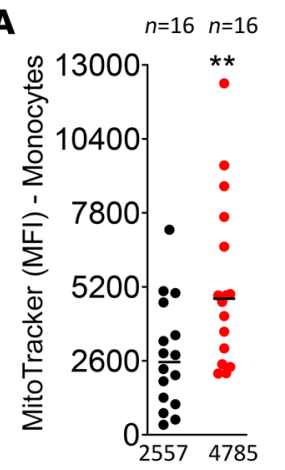

ICU patients without sepsis
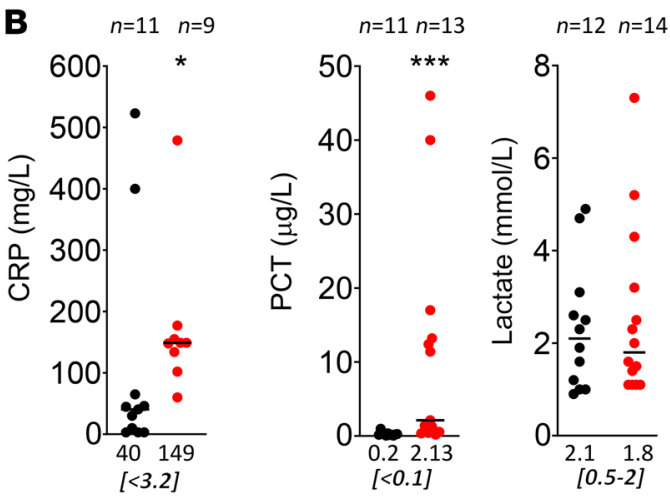

ICU patients with sepsis

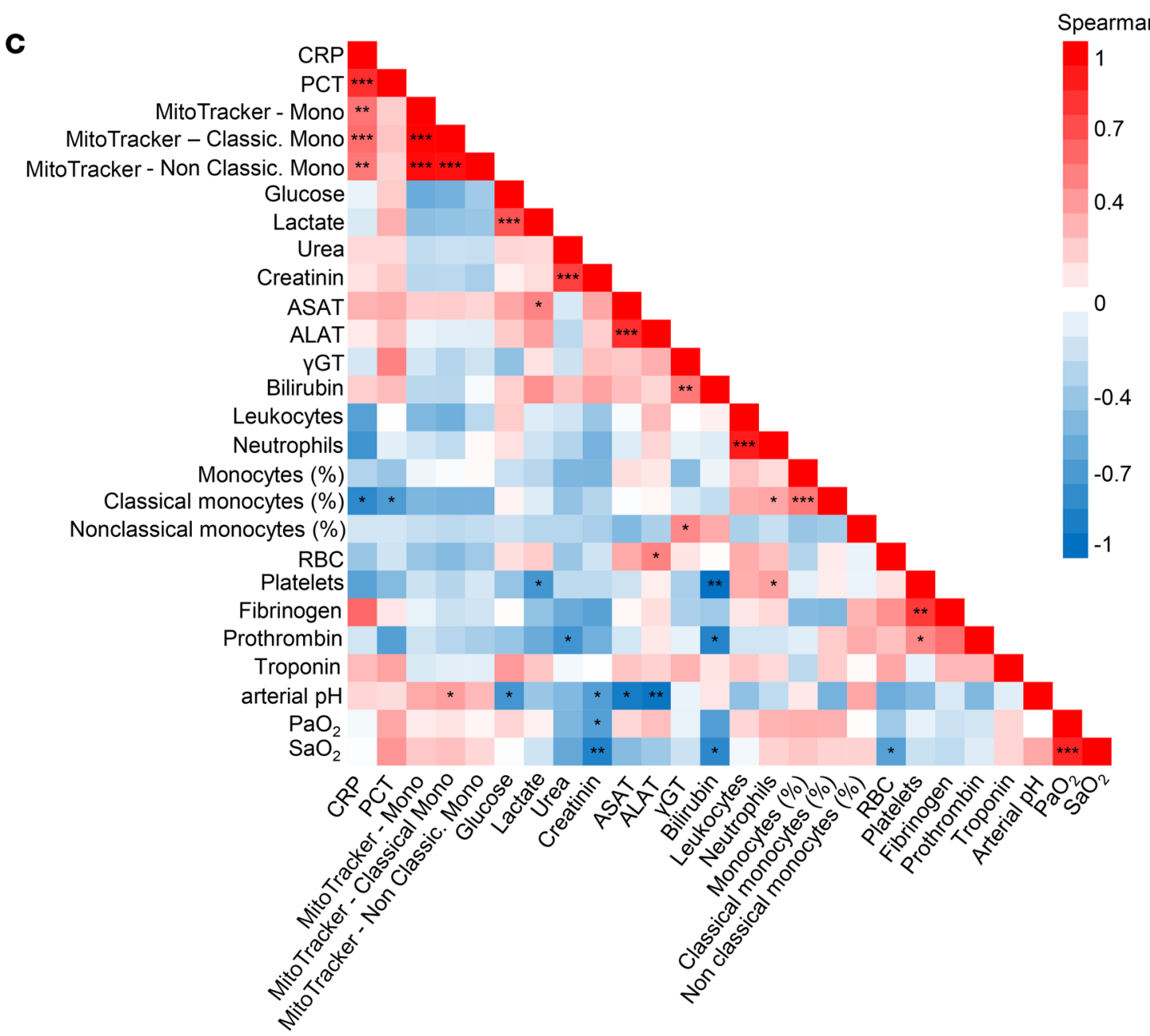

Figure 8. Increased mitochondrial density in blood monocytes is a biomarker of sepsis in critically ill patients. (A) Flow cytometry assessment of mitochondrial density in blood monocytes of critically ill patients (ICU patients without [ $n=16$ ] or with sepsis [ $n=16]$ according to sepsis-3 task force criteria). (B) Levels of inflammatory and tissue perfusion biomarkers in the blood of critically ill patients as described in $\mathbf{A}$. Data were collected from patient medical records and were not available for all patients ( $n$ indicated above graphs). The graphs represent median plus individual values. The median value of each group is presented at the bottom of each graph. The normal range of healthy patients is presented in bold italics enclosed in square brackets. ${ }^{*} P<0.05$, ${ }^{* *} P<0.01,{ }^{* *} P<0.001$ determined by Student's $t$ test with Welch's correction. (C) Correlation matrix of blood biomarkers in critically ill patients as described in $\mathbf{A}(n=15-32) .{ }^{*} P<0.05,{ }^{* *} P<0.01,{ }^{* *} P<0.001$ were determined by Spearman's rank correlation.

was more pronounced when caspase- 4 was incubated with LPS (Supplemental Figure 5F). Unexpectedly, a similar observation was made for caspase-1 (Figure $5 \mathrm{E}$ and Supplemental Figure $5 F$ ). Beyond 1-hour incubation with recombinant caspase-4,
Tribolium castaneum PINK1 was not detectable anymore (data not shown). In contrast to LPS, zVAD-FMK treatment reduced caspase-mediated PINK1 degradation (Figure 5E and Supplemental Figure 5F). 
LPS/IFN-y-mediated inhibition of mitophagy triggers classical activation of macrophages through mROS. The inhibition of mitophagy is a critical source of mROS (ref. 14, Figure 1B, and Supplemental Figure 1B). Since we showed that the inhibition of mitophagy was an early feature of macrophage activation, we examined whether mROS could directly trigger the classical activation of macrophages. Mitochondrial CI and CIII, whose activities were reduced in LPS/IFN- $\gamma$-exposed raw 264.7 macrophages (Supplemental Figure 6A), are 2 major sources of mROS $(14,48)$. We thus used rotenone and antimycin A, respective CI and CIII inhibitors, to promote mROS production (Supplemental Figure 6B and ref. 14). However, only CIII inhibition efficiently promoted an increase in macrophage activation markers in raw 264.7 (Figure 6A and Supplemental Figure 6C). This parallels the increase in $\mathrm{mROS}$, which was more pronounced with antimycin A compared with rotenone (Supplemental Figure 6B). Along with raw 264.7 macrophage activation, antimycin A triggered the release of inflammatory cytokines (Supplemental Figure 6E). The exposure of BMDMs to MitoParaquat, an mROS generator, further strengthened the role of mROS in macrophage activation (Supplemental Figure 6D). Moreover, scavenging of mROS induced by LPS/IFN- $\gamma$ with mitoTEMPOL, an mROS scavenger, or with N-acetyl-L-cysteine (NAC), a general ROS scavenger, decreased raw 264.7 macrophage activation (Figure 6B and Supplemental Figure 6F). Accordingly, ROS scavengers also prevented LPS/IFN- $\gamma$-mediated inflammatory cytokine release and phagocytosis of latex beads in raw 264.7 macrophages (Supplemental Figure 6, G and H).

Inhibition of mitophagy induces the classical activation of macrophages through HIF- $1 \alpha$. HIF- $1 \alpha$ contributes to the functional reprogramming of monocytes during sepsis (49) and to metabolic reprogramming during macrophage activation where it can be activated and stabilized by succinate or mROS $(48,50$, 51). Accordingly, HIF-1 $\alpha$ protein levels were increased by both LPS/IFN- $\gamma$ and mdivi-1 exposure in raw 264.7 macrophages (Supplemental Figure 6K). Interestingly, echinomycin, a pharmacological inhibitor of HIF-1 $\alpha$ (52), blunted the activation of raw 264.7 macrophages triggered by LPS/IFN- $\gamma$, antimycin A, MitoParaquat, and mdivi-1 (Figure 6C and Supplemental Figure $6, \mathrm{D}, \mathrm{I}$, and J). In agreement with the contribution of mROS to macrophage activation, we showed that mdivi-1 and other mitophagy inhibitors promoted classical activation of raw $264.7 \mathrm{mac}-$ rophages, as assessed by flow cytometry or by gene expression (Figure 6, D and E, and Supplemental Figure 7, A and B). The inhibition of mitophagy in raw 264.7 macrophages accompanied elevated inflammatory cytokine release and increased phagocytosis of latex beads (Supplemental Figure 7, C and D). In line with their ability to commit raw 264.7 macrophages toward an inflammatory phenotype, pharmacological inhibitors of mitophagy boosted their bactericidal activity (Figure 6F and Supplemental Figure 7E). In contrast to mdivi-1, exposure of activated raw 264.7 macrophages to the mitochondrial uncouplers CCCP and 2,4-DNP promoted mitophagy and reversed LPS/IFN- $\gamma-$ mediated inhibition of mitophagy (Figure $1 \mathrm{G}$ and Supplemental Figure 1F). This translated into a reduction of raw 264.7 macrophage activation and bactericidal activity (Figure 6, G-I, and Supplemental Figure 7, F and G).
Inhibition of mitophagy in myeloid cells protects against bacterial infection. We were able to translate our results in vivo in mice because the administration of mitochondrial uncouplers alone or in combination with LPS in mice mirrored our in vitro observations. The induction of mitophagy was associated with reduced mitochondrial density in peritoneal macrophages. More strikingly, LPS-mediated inhibition of mitophagy in peritoneal macrophages was reversed by CCCP and 2,4-DNP administration (Supplemental Figure 8, A and B). Then, we used a single i.p. injection of 2,4-DNP (10 mg/ $\mathrm{kg}$ ) to promote mitophagy 24 hours before CLP surgery. Whereas 2,4-DNP did not affect the survival of animals receiving the sham operation, it significantly reduced the survival of animals receiving CLP (Figure 7A). As expected, the mitochondrial density of peritoneal macrophages was reduced as a consequence of 2,4-DNP-induced mitophagy (Supplemental Figure 8C). This stimulation of mitophagy translated into a decrease in macrophage activation, as illustrated by the reduction of the proportion of $\mathrm{CD} 80^{\text {hi }} \mathrm{CD} 64^{\text {hi }}$ macrophages and lowered levels of plasma inflammatory cytokines (Figure 7B and Supplemental Figure 8, D and G). The total number of peritoneal macrophages was not altered by 2,4-DNP (Supplemental Figure $8 \mathrm{E}$ ). This dampened macrophage activation resulted in a higher bacterial load in the peritoneal cavity of 2,4-DNP-injected animals (Figure 7C), with a similar trend in the liver (Supplemental Figure $8 \mathrm{~F}$ ). No changes in blood bacteremia were observed (data not shown). We ranked peritoneal bacterial load above $1.10^{6}$ $\mathrm{CFU} / \mathrm{mL}$ as high bacterial load and noticed that animals with the highest bacterial load presented a significant reduction in the proportion of CD $64^{\text {hi }}$ Mitotracker ${ }^{\text {hi }}$ macrophages in the peritoneal cavity (Supplemental Figure 8H).

Early inhibition of mitophagy in myeloid cells is a predictive factor of survival upon sepsis. This led us to assume that the inhibition of mitophagy is protective against bacterial invasion during sepsis. In line with this, we found a significant positive correlation between mitochondrial density in Ly6 $\mathrm{C}^{\text {hi }}$ blood monocytes collected 2 hours after CLP surgery and the survival of animals over a period of 10 days (Figure 7D). More expected, the percentage of Ly6C $\mathrm{C}^{\text {hi }}$ blood monocytes at 2 hours was also correlated with mouse survival (Supplemental Figure 8J), but the percentage of Ly6 $\mathrm{C}^{\text {hi }}$ blood monocytes and mitochondrial density in these monocytes were not correlated (Supplemental Figure 8K). Therefore, mitochondrial density in Ly6C ${ }^{\text {hi }}$ blood monocytes at an early time point after polymicrobial infection was an independent predictor of survival. Finally, we sorted animals into short-term ( $<72$ hours survival) and long-term ( $>72$ hours survival) survivors and showed that relative to short-term survivors, long-term survivors had higher mitochondrial density (mitophagy inhibition) in Ly6 $\mathrm{C}^{\text {hi }}$ blood monocytes (Supplemental Figure 8I).

Inhibition of mitophagy in myeloid cells reduces bacterial load upon sepsis. To further confirm the protective role of mitophagy inhibition in myeloid cells against polymicrobial infection, we i.p. injected C57BL6/J mice with mdivi-1 (50 mg/kg) 24 hours before sham or CLP surgery. The i.p. injection of mdivi-1 in mice mimicked the increase in the proportion of $\mathrm{CD} 64^{\mathrm{hi}}$ Mitotracker ${ }^{\text {hi }}$ peritoneal macrophages observed in mice receiving CLP or LPS (Supplemental Figure 9A). Moreover, in accordance with the inhibition of mitophagy promoted by mivi-1, mitochondrial density was also increased 
in Ly6C $\mathrm{C}^{\text {hi }}$ blood monocytes (Supplemental Figure 9B). In contrast to 2,4-DNP-induced mitophagy, the i.p. injection of mdivi-1 24 hours before CLP surgery led to a significant increase in the survival of mdivi-1-treated mice (Figure 7E). The increased proportion of activated macrophages in the peritoneal cavity (Figure 7F) contributed to the reduced bacterial load observed in the peritoneal cavity (Figure $7 G$ ), the liver, and the blood of mdivi-1-injected mice (Supplemental Figure 9, C and D) and their improved survival compared with vehicle-treated mice. It is noteworthy that the proportion of CD64 ${ }^{\text {hi }}$ Mitotracker ${ }^{\text {hi }}$ peritoneal macrophages was also higher in mdivi-1-treated mice that underwent CLP surgery (Supplemental Figure 9E), whereas the percentage of total peritoneal macrophages was not affected by mdivi-1 pretreatment (Supplemental Figure 9F). Because our in vitro data argued in favor of a key role of PINK1-dependent mitophagy in the LPS-dependent activation of myeloid cells, we performed transplantation of $\mathrm{Pink1}^{+/+}$or $\mathrm{Pink1}^{-/-}$bone marrow in C57BL6/J recipient mice (bone marrow transplant [BMT] Pink $1^{+/}$, BMT Pink $1^{--}$). After 5 weeks of recovery, chimeric mice with Pink1-hematopoietic deficiency and their control counterparts that received $\mathrm{PINK}^{+/+}$bone marrow underwent CLP surgery (Figure $7 \mathrm{H})$. Similar to the pharmacological inhibition of mitophagy, hematopoietic deficiency in Pink1 led to an increase in mitochondrial density in peritoneal macrophages (Supplemental Figure 9G). More spectacularly, survival after CLP was improved in mice transplanted with Pink1 $1^{-/}$bone marrow (Figure 7H). This can be attributed, at least in part, to the increased proportion of activated macrophages (Figure 7I) and of CD64 ${ }^{\text {hi }}$ /Mitotracker ${ }^{\text {hi }}$ macrophages (Supplemental Figure 9H) in the peritoneal cavity, which contributed to a lower bacterial load in the peritoneal cavity (Figure 7J) and in the liver (Supplemental Figure 9I). It is noteworthy that the percentage of peritoneal macrophages did not differ between $\mathrm{Pink1}^{-{ }^{-}}$and $\mathrm{Pink1}^{+/+}$ chimeric mice (Supplemental Figure 9J).

Inhibition of mitophagy in blood monocytes is a marker of sepsis in critically ill patients. Finally, we aimed to assess the clinical relevance of our in vitro and in vivo findings in mice. Mitochondrial density was assessed in blood monocytes of 32 critically ill patients within 8 hours after their admission to the ICU. Blood markers of inflammation, metabolism, liver function, renal function, cardiac function, hemodynamics, and tissue perfusion monitored within the same timeframe were collected from the medical records. The causes of admission as well as age and gender distribution of the patients are depicted in Supplemental Table 1 (median age: 59.5 years nonseptic patients vs. 62 years septic patients, NS, $P$ $=0.55$ ). Sixteen patients presented with sepsis according to the definition of the sepsis-3 task force (53). Mitochondrial density in blood monocytes was significantly higher in septic patients compared with nonseptic patients (Figure 8A). A similar observation was made in classical and nonclassical monocyte subpopulations (Supplemental Figure 10B). Of note, the percentage of monocytes was not significantly different between the 2 groups of patients, despite a trend to be lower in septic patients (median: 9.2\% nonseptic vs. $4.8 \%$ septic) (Supplemental Figure 10C). The percentage of classical monocytes $\left(\mathrm{CD} 14^{++} \mathrm{CD} 16^{-}\right)$was significantly reduced in septic patients (median: $83.7 \%$ of total monocytes nonseptic vs. $68.9 \%$ septic) (Supplemental Figure 10C). Nonclassical (inflammatory) monocytes and intermediate monocytes displayed a nonsignificant trend toward an increase (Supplemental Figure 10C).
Inflammatory markers, namely $\mathrm{C}$-reactive protein (CRP) and procalcitonin (PCT), have a pivotal role in the diagnosis of sepsis. Both were above the normal range in all ICU patients and, as expected, were at significantly higher levels in septic patients (Figure 8B). PCT, as a marker of severe infection, was particularly increased in septic patients, who had a 10-fold higher median value compared with nonseptic patients (Figure 8B). Other inflammatory markers such as leukocyte count and neutrophil count were not significantly different between the 2 groups (Supplemental Figure 10D). Lactate levels are an indicator of tissue perfusion used as a criterion of septic shock in patients with sepsis (53). Here, blood lactate levels did not allow differentiation between the groups of patients (Figure $8 \mathrm{~B}$ ). The median of blood markers of renal function (Supplemental Figure 10E), metabolism (Supplemental Figure 10F), liver function (Supplemental Figure 10G), coagulation (Supplemental Figure $10 \mathrm{H})$, cardiac function (Supplemental Figure 10I), tissue oxygenation, and hemodynamics (Supplemental Figure 10J) were almost all out of the normal range (normal range of healthy patients is presented in bold italics enclosed in square brackets) for both groups of critically ill patients. With the exception of arterial $\mathrm{pH}$, none of the recorded parameters allowed differentiation between septic and nonseptic patients. Therefore, mitochondrial density in monocytes arose as a potential biomarker of sepsis in critically ill patients, similar to CRP and PCT. Because of high variation among patients, we also analyzed the correlation between all these monitored parameters (Figure $8 \mathrm{C}$ ). Only a few parameters were significantly correlated. As expected, CRP and PCT were highly correlated $(r=0.82$, $P=0.0003$ ) (Figure 8C). Interestingly, similar to PCT, mitochondrial density in total monocytes $(r=0.56, P=0.01)$, as well as classical $(r=0.59, P=0.006)$ and inflammatory monocytes $(r=0.54$, $P=0.01$ ), were positively correlated to CRP (Figure $8 \mathrm{C}$ ). These data further suggest that mitophagy levels in blood monocytes could be a useful marker for sepsis in critically ill patients. Further studies are required to assess whether this parameter is differentially altered according to sepsis severity or survival.

\section{Discussion}

Altogether, our observations showed that the early inhibition of mitophagy in myeloid cells contributed to their activation and improved the outcome of gram-negative bacterial infections and septic conditions in mice. The translational value of these findings was documented, and the mitophagy level in blood monocytes was highlighted as a biomarker of sepsis in critically ill patients.

The current view is that the induction of mitophagy protects against sepsis, based on the observation that altered mitochondrial function leads to excessive mROS production, the release of mtDNA, and the activation of the NLRP3 inflammasome $(14,15$, $54)$. Nevertheless, the contribution of mROS to sepsis is controversial. mROS leads to NLRP3 activation, which is associated with IL-1 $\beta$ release and reduced survival $(14,40)$. However, in macrophages, mROS production contributes to bacterial clearance and host defense $(26,27)$. In addition, mROS scavenging with SkQ1 or MitoTEMPOL in CLP-mediated polymicrobial infection in mice significantly reduced survival (55). Our data demonstrating that LPS/IFN- $\gamma$-mediated inhibition of mitophagy in macrophages led to enhanced mROS production and triggered the activation of macrophages are in line with these observations. 
Classically, mitophagy is assessed by Western blotting and microscopy. Such strategies are not easily applicable to macrophages in vivo at early time points after bacterial infection. To overcome this technical issue, we first showed that the assessment of mitochondrial density by flow cytometry is a reliable marker of mitophagy. Then, we transposed this approach to freshly collected blood and peritoneal fluid in mice and critically ill patients. This cytometry approach allowed us to track mitophagy extemporaneously in subsets of immune cells extemporaneously as early as 1 hour after infection. Whereas our study focused on early time points, most previous studies that investigated mitophagy in the context of sepsis considered time points beyond 24 hours. Earlier reports described an induction of mitophagy during sepsis (13, $56,57)$. Our data do not oppose such previous studies, but rather bridge a gap by focusing on early stages of macrophage activation. In vitro, we observed that LPS/IFN- $\gamma$-exposed BMDMs displayed a drop in the protein levels of mitophagy checkpoints. After this initial decrease, we noticed an increase in PINK1, Parkin, and DRP1 protein levels in BMDMs exposed to LPS/IFN- $\gamma$ over 18 hours (data not shown). Therefore, after an initial decrease upon macrophage activation, mitophagy was reactivated in response to the deleterious impact of a perpetuated inhibition of mitophagy.

Our study also portrayed the metabolic shifts associated with macrophage activation and LPS/IFN- $\gamma$-mediated inhibition of mitophagy. A drop in the $\Delta \psi \mathrm{m}$ is a well-characterized trigger that initiates PINK1-dependent mitophagy $(17,31)$. In line with this, we found that LPS/IFN- $\gamma$-mediated inhibition of mitophagy in macrophages was linked to a rise in $\Delta \psi \mathrm{m}$ with hyperfused mitochondria. Classically, such a phenotype is related to enhanced OXPHOS $(6,58)$. Nevertheless, in LPS/IFN- $\gamma$-activated macrophages, mitochondrial respiration drops, whereas extracellular acidification increases. This underlies their glycolytic shift (6-8). Of note, this metabolic profile is a feature of LPS/IFN- $\gamma$-activated macrophages in that the activation state triggered by the TLR2 ligand Pam3CysSK4 is associated with a different metabolic signature characterized by increased ECAR and oxygen consumption (32). Interestingly, by inhibiting mitophagy with mdivi-1, we observed a metabolic rewiring similar to that observed in LPS/ IFN- $\gamma$-activated macrophages. Similarly, Pink 1 deficiency is associated with a fall in mitochondrial respiration; a reduction in $\mathrm{CI}$, CIII, and CIV activity; and a metabolic reprogramming toward glycolysis (59, 60). Along with these changes, Pink1-deficient myocytes exhibit a pronounced increase in $\Delta \psi \mathrm{m}$ compared with WT myocytes (61), which further consolidates the idea that mitophagy inhibition contributes to the metabolic rewiring associated with macrophage activation. In Pink1-deficient mouse embryonic fibroblasts, the maintenance of cell viability involves HIF-1 $\alpha$ subsequent to its activation by mROS (60). In our settings, we showed that mROS and HIF-1 $\alpha$ also had a pivotal role in macrophage activation after LPS/IFN- $\gamma$-mediated mitophagy inhibition.

Whereas mROS can be generated by the activation of various TLR agonists (26), we showed that upon macrophage activation, mROS generated in a mitophagy-dependent manner were exclusively triggered by LPS. In addition to TLR4, STAT1 signaling holds a central position in LPS/IFN- $\gamma$-mediated macrophage activation $(4,62)$. We demonstrated that STAT1 was directly involved in LPS/IFN- $\gamma$-mediated inhibition of mitophagy. Interestingly, a link between STAT1 and mitochondrial function was previously suggested (63-65). Our work also showed that the activation of STAT1 signaling resulted in the inhibition of mitophagy in a caspase-1- and caspase-11-dependent manner. These interconnected inflammatory caspases are involved in LPS-dependent activation of the NLRP3 inflammasome, pyroptosis, and IL-1 $\beta$ release $(40,66)$. Upon activation of the NLRP3 inflammasome, caspase-1 was shown to translocate to mitochondria to block mitophagy by degrading Parkin $(37,38)$. Here, we showed that the pancaspase inhibitor z-VAD or siRNA targeting caspase-1 abrogated LPS/IFN- $\gamma$-mediated downregulation of PINK1 protein levels and subsequently inhibited mitophagy. Because caspase- 1 and caspase-11 have related functions, we also investigated them. We discovered common functions with regards to LPS/IFN- $\gamma$-mediated mitophagy inhibition. First, we found that both caspase- 1 and -11 are STAT1 target genes. Whereas STAT1-dependent regulation of caspase-1 was unknown, the levels of caspase-11 were previously shown to be upregulated by IFN- $\gamma$, but without demonstration of STAT1 involvement (39). Additionally, similar to caspase-1, we showed that caspase-11 translocated to mitochondria upon LPS/ IFN- $\gamma$-mediated macrophage activation. More interestingly, using recombinant proteins, we showed that both caspase- 1 and -11 were able to degrade PINK1. A similar property was previously depicted for caspase-11 toward TRPC1 (42). Besides this action, caspase-11 was shown to act as an intracellular sensor binding LPS $(46,47)$. Interestingly, the presence of LPS markedly increased the propensity of caspase-11 for degrading PINK1. Unexpectedly, we made a similar observation for caspase-1. This further strengthens the hypothesis of coordinated action of these 2 inflammatory caspases to block mitophagy by degrading PINK1 upon STAT1 activation and in the presence of LPS intracellularly.

We studied the scope of these in vitro findings in vivo in murine models of polymicrobial infection (CLP) and endotoxemia (LPS injection). We showed that with pharmacological modulators of mitophagy, as well as with transplantation of Pink1-deficient bone marrow, the inhibition of mitophagy in myeloid cells led to their activation, contributed to the reduction of bacterial infection, and was associated with a better survival. These data also raise preliminary evidence of a potential therapeutic interest of pharmacological targeting of mitophagy in myeloid cells of septic patients. We showed that the early measurement of mitochondrial density in blood monocytes by flow cytometry upon the onset of sepsis was a reliable marker of mitophagy level, and this parameter was assessed in critically ill patients hospitalized in the ICU with or without sepsis. Interestingly, we observed that mitochondrial density in blood monocytes (a marker of mitophagy inhibition) was significantly increased in septic patients compared with nonseptic patients. Similar to blood PCT, a biomarker of severe infection classically used in the diagnosis of sepsis, mitochondrial density in blood monocytes was significantly correlated with blood level of CRP, and only with this parameter, among 20 blood biomarkers (inflammation, organ dysfunction, tissue perfusion, hemodynamics). Therefore, we propose the mitophagy level in blood monocytes as a biomarker of sepsis. Future studies will be required to investigate the level of mitophagy in blood monocytes according to sepsis severity, survival, and type of infection in order to evaluate whether this parameter could be helpful in personal- 
ized patient care. Regarding the type of infection, our in vitro and in vivo findings in mice showed that the inhibition of mitophagy in myeloid cells was a feature of LPS and gram-negative infections. Among the set of critically ill patients studied, sepsis was not likely related to gram-negative infection in all cases. Nevertheless, the contribution of LPS in the inhibition of mitophagy in blood monocytes of critically ill patients cannot be excluded: our group recently showed, using a new highly sensitive mass spectrometry method, that blood levels of LPS are significantly higher in septic versus nonseptic patients whatever the type of infection (67). This phenomenon is likely due to the translocation of gram-negative bacteria because of the alteration of barrier functions in septic patients without regard to the primary infection. This will have to be studied with respect to the mitophagy level in the blood monocytes of critically ill patients. Finally, this work raises the hypothesis of the use of pharmacological modulators of mitophagy in septic patients. This strategy may enhance host defense and reverse sepsis-induced immunoparalysis. Additionally, our data suggest that therapeutics endowed with mitophagy-modulating properties should be investigated in the context of septic patients. Such investigations should, in particular, explore the impact of antibiotics because recent studies have highlighted important consequences of several classes of antibiotics on mitochondrial function (68). This may help in understanding some unexplained therapeutic failures with certain antibiotics.

\section{Methods}

Reagents and antibodies. See Supplemental Methods.

Bacterial load. Peritoneal fluids and liver homogenates were subjected to serial 10 -fold dilutions and cultured on $5 \%$ sheep blood agar (Oxoid) plates. CFUs were quantified after 24 hours of incubation at $37^{\circ} \mathrm{C}$ in anaerobic conditions.

Recombinant protein assay. Recombinant proteins were stored at $-80^{\circ} \mathrm{C}$ until used in PBS containing $15 \%$ glycerol. Recombinant $\mathrm{T}$. Castaneum PINK1 (260 ng) was incubated at $37^{\circ} \mathrm{C}$ with recombinant human caspase-1 (1 U) or caspase-4/11 (1 U) (Enzo Life Sciences) for the indicated duration in $50 \mu \mathrm{L}$ assay buffer (50 mM HEPES pH 7.2, $50 \mathrm{mM} \mathrm{NaCl}, 0.1 \%$ CHAPS (3-[(3-Cholamidopropyl)dimethylammonio]-1-propanesulfonate hydrate), 10 mM EDTA, 5\% glycerol, 10 $\mathrm{mM}$ DTT). Then, proteins were denatured for 5 minutes at $100^{\circ} \mathrm{C}$ in Laemmli buffer and loaded on 2,2,2-TCE gel (mini protean Tris-Glycine eXtended, stain-free, 4\%-20\% from Bio-Rad Laboratories). Proteins were visualized by UV transillumination.

Cellular studies. Raw 264.7 cells were obtained from ATCC (TIB71) and cultured in RPMI 1640 medium containing L-glutamine supplemented with $10 \%$ FBS plus antibiotics $(100 \mu \mathrm{g} / \mathrm{mL}$ penicillin, 100 $\mu \mathrm{g} / \mathrm{mL}$ streptomycin). Murine BMDMs were generated from C57BL/6J mice and cultured in RPMI 1640 medium containing L-glutamine supplemented with 10\% FBS, 20\% L929-conditioned media, and antibiotics. Peritoneal macrophages were harvested from C57BL/6J mice by puncture of the peritoneal cavity after injection of $5 \mathrm{~mL}$ of cold PBS 2 mM EDTA (no thioglycolate elicitation). Peritoneal macrophages were allowed to attach for 12 hours (raw 264.7 cell medium). The medium was renewed after this period.

In vitro cell stimulation. See Supplemental Methods.

OCR and ECAR. Real-time OCR and ECAR in BMDMs were determined with a Seahorse XF96e Extracellular Flux Analyzer (Agi- lent Technologies). For each condition, $4 \times 10^{4}$ cells/well in 6 to 8 wells were used. The assay was performed in DMEM-based medium according to the manufacturer's instructions. Four consecutive measurements were performed under basal conditions and 3 after the sequential addition of the following respiratory chain complex inhibitors (Mito Stress assay [oligomycin, FCCP, antimycin A/rotenone]; Agilent Technologies). OCR and ECAR data were normalized per microgram of protein per well.

Cytokine quantification. Cytokines were measured at the indicated time points on cell culture media or mouse plasma according to the manufacturer's instructions for Luminex-based multiplex assays (Merck-Millipore).

Macrophage activation. Cells were suspended in PBS 2 mM EDTA. $\mathrm{Fc} \gamma$ receptors were saturated with FcR blocking reagent (Miltenyi Biotec) according to the manufacturer's instructions. After a washing step, cells were stained with anti-mouse CD64-APC, anti-mouse CD80-PE, or anti-mouse CD86-PE antibodies according to the manufacturer's instructions (Miltenyi Biotec). Cells were maintained on ice until flow cytometry analysis.

Mitochondrial density. For in vitro studies, after stimulation, cells were incubated for 20 minutes at $37^{\circ} \mathrm{C}$ in a humidified $\mathrm{CO}_{2}$ incubator with preheated PBS containing 200 nM MitoTracker Green FM (Cell Signaling). Cells were rinsed and resuspended in PBS $2 \mathrm{mM}$ EDTA. Cells were maintained on ice until flow cytometry analysis. For in vivo studies, hemolysis was performed on freshly collected blood samples or leukocytes obtained from peritoneal fluid. After rinsing, cells were incubated with PBS containing 200 nM MitoTracker Green FM for 20 minutes and saturated with FcR blocking reagent (Miltenyi Biotec) according to the manufacturer's instructions. After a washing step, cells were stained with anti-mouse CD45-Viogreen; CD115-PE; Ly6G-VioBlue; Ly6C-APC; CD64-APC; CD80-PE; CD86-PE; F4/80PerCP-Vio700; and CD11b-APC-Vio770 (Miltenyi Biotec). Flow cytometry analysis was performed with a BD LSR II flow cytometer (BD Biosciences). For human studies, a similar procedure was used. After hemolysis and rinsing, cells were incubated with PBS containing 200 nM MitoTracker Green FM for 20 minutes. After a washing step, cells were placed on ice and stained with anti-human CD33-PEVio770 and CD66b-APC-Vio770 (Miltenyi Biotec), anti-human CD14BV 605 and CD16-BV 421 (BD Biosciences). Flow cytometry analysis was performed with a BD FACS Canto Cytometer (BD Biosciences).

Mitochondrial ROS production and $\triangle \psi m$. After stimulation, cells were incubated for 20 minutes at $37^{\circ} \mathrm{C}$ in a humidified $\mathrm{CO}_{2}$ incubator with preheated PBS containing $2.5 \mu \mathrm{M}$ MitoSOX red (Thermo Fisher Scientific) for mROS or $5 \mu \mathrm{M} \mathrm{JC}-1$ or $50 \mathrm{nM}$ TMRM (tetramethylrhodamine, methyl ester, perchlorate; Thermo Fisher Scientific) for $\Delta \psi \mathrm{m}$. Cells were rinsed, resuspended in PBS $2 \mathrm{mM}$ EDTA, and analyzed by flow cytometry.

Flow cytometry assessment of autophagy and mitophagy. After stimulation, cells were stained with CYTO-ID Autophagy Detection Kit (Enzo Life Sciences) according to the manufacturer's instructions. Flow cytometry analysis was performed with a BD LSR II flow cytometer. For flow cytometry assessment of mitophagy, raw 264.7 cells were transfected with mitochondrial mKeima (mt-mKeima) expression vector or empty vector using JetPEI macrophages (PolyPlus Transfection) according to the manufacturer's instructions. Then, 24 hours after the transfection, the medium was changed and cells were exposed to LPS/ IFN- $\gamma$ or vehicle for 6 hours or 24 hours. The spectral shift of mitochondrial-targeted mt-mKeima was assessed by flow cytometry using 
a BD LSRII flow cytometer. During mitophagy, mKeima expressed in mitochondria that are engulfed into lysosomes are excited at $561 \mathrm{~nm}$ because of low lysosomal $\mathrm{pH}$. In mitochondria that are not committed to mitophagy, mKeima is maintained in a neutral $\mathrm{pH}$ environment and emits a signal when excited at $406 \mathrm{~nm}$ (69).

Gentamycin protection assay. Raw 264.7 macrophages were exposed to 3-MA, 2,4-DNP, or CCCP only before the gentamycin protection assay. E. coli-GFP were added at an MOI of 10 into the medium of raw 264.7 cells and incubated for 1 hour at $37^{\circ} \mathrm{C}$. Medium was then refreshed with medium containing gentamycin in order to eliminate nonphagocytized E. coli-GFP. Phagocytosis and intracellular lysis E. coli-GFP were followed by flow cytometry (see Supplemental Methods).

Phagocytosis assays. Cells were stimulated and incubated at $37^{\circ} \mathrm{C}$ with carboxylate-modified polystyrene and fluorescent red latex beads ( $\sim 2 \mu$ m diameter) (Sigma-Aldrich) at a ratio of 50 beads/cell in the culture medium. After the 18-hour incubation, cells were washed with ice-cold PBS and analyzed by flow cytometry.

Flow cytometry analysis. Unless specified, flow cytometry was performed with a BD LSR II flow cytometer. All flow cytometry data were then analyzed with FlowJo v10 software.

Mitochondria isolation. Mitochondria were isolated with a mitochondria isolation kit using anti-Tom22 antibodies (all from Miltenyi Biotec) coupled with magnetic microbeads, according to the manufacturer's instructions.

Western blot analysis. See Supplemental Methods.

Microscopy. For JC-1 and MitoTracker Green staining and cells transfected with pMito-LSSmOrange (OriGene) or empty vector, cells were plated on glass coverslips. After stimulation, glass coverslips were mounted in cold PBS and directly visualized with Axio imager 2 (Carl Zeiss Microscopy $\mathrm{GmbH}$ ). For immunostaining, cells were grown on Labtek slides. After stimulation, cells were fixed with $4 \%$ paraformaldehyde by incubating for 10 minutes at $4^{\circ} \mathrm{C}$. Cells were then permeabilized by incubating with PBS containing 0.1\% Triton X-100. Blocking was carried out with PBST (PBS + 0.1\% Tween-20) supplemented with $1 \%$ BSA and $10 \%$ goat serum at $4^{\circ} \mathrm{C}$ for 30 minutes. Cells were then incubated with primary antibody at $4^{\circ} \mathrm{C}$ overnight in a humidified chamber. Cells were then washed and incubated with specific secondary antibody conjugated with a fluorophore for 1 hour under humidified chamber in the dark. Preparations were visualized with a Leica SP2 confocal microscope. Images were analyzed with ImageJ software (NIH).

Quantitative PCR. See Supplemental Methods.

Oxygen consumption. After stimulation, $4 \times 10^{6}$ cells were resuspended in $1 \mathrm{~mL}$ of preheated $\mathrm{PBS}$ at $37^{\circ} \mathrm{C}$. Oxygen consumption was measured with Clark electrodes.

Respiratory chain complex activity. Cell pellets were homogenized, and enzyme activities were measured by spectrophotometric methods as previously described (70).

Mouse studies. C57BL/6 mice were obtained from Charles River Laboratories. Pink1 $1^{-/}$mice and control littermates were obtained from The Jackson Laboratory. Animals were housed in a temperature- and humidity-controlled facility and fed a standard chow diet. Experiments were performed with age-matched female mice (8-12 weeks old). CLP surgery was carried out as previously described (71). Blood sampling was performed by a retroorbital puncture in mice anesthetized with isoflurane. Peritoneal washing was performed after euthanasia. Next, $5 \mathrm{~mL}$ of $2 \mathrm{mM}$ EDTA PBS were i.p. injected and recovered by peritoneal puncture. Endotoxemia was induced by i.p. injection of LPS (E. coli serotype O55:B5) administered at 0.5 or $15 \mathrm{mg} / \mathrm{kg}^{-1}$ for 24 hours. 2,4-DNP was prepared in PBS and given by i.p. injection (10 $\mathrm{mg} / \mathrm{kg}^{-1}$, single dose) 24 hours prior to sham or CLP surgery or LPS injection. In vivo toxicity of 2,4-DNP has been extensively documented, with an $\mathrm{LD}_{50}$ of $72 \mathrm{mg} / \mathrm{kg}$ reported in mice (72). Mdivi-1 (BioTechne) was diluted in sterile saline (1\% DMSO) and gently sonicated before i.p. injection $(50 \mathrm{mg} / \mathrm{kg})$ to obtain a homogenous suspension. Control mice received vehicle only ( $5 \mathrm{~mL} / \mathrm{kg}$, PBS plus 1\% DMSO).

Bone marrow transplantation. Eight-week-old female C57BL6/J recipient mice were irradiated with 11 Gy to trigger full medullary aplasia before transplantation. A suspension of bone marrow cells was prepared from femurs of 6-week-old male $\mathrm{Pink1}^{+/+}$or $\mathrm{Pink1}^{-/-}$mice. Recipient mice were injected with about $2 \times 10^{6}$ bone marrow cells through the tail vein. C57BL6/J recipient mice were given water containing enrofloxacin $5 \mathrm{mg} / \mathrm{kg}$ for 2 weeks after transplantation. CLP was performed on chimeric mice after 5 weeks of recovery after BMT.

Human studies. Critically ill patients aged 18 years or older admitted to the ICU of University Hospital François Mitterrand, Dijon, France, receiving at least 1 life support therapy for organ failure (e.g., mechanical ventilation, vasopressors or inotropic agents, renal replacement therapy, high-flow nasal cannula) with or without sepsis were eligible for inclusion (observational study Myelochondria). Baseline severity of illness was assessed using the Simplified Acute Physiology Score (SAPS II), and the degree of baseline organ dysfunction was quantified using the Sequential Organ Failure Assessment (SOFA) score. Patients were prospectively classified into subgroups according to the sepsis-3 definition (53). Only blood samples collected on EDTA-coated tubes for routine hematology analysis $(<8$ hours since admission to ICU) were used for flow cytometry analysis of mitochondrial density in blood monocytes. Biological parameters obtained within a similar timeframe were recovered from the medical records.

Statistics. Statistical differences were analyzed with GraphPad Prism 8. Comparisons of 2 groups were calculated with unpaired Student's $t$ test with Welch's correction. For comparisons with more than 2 groups, Brown-Forsythe and Welch ANOVA tests were used. Correction for multiple comparisons was performed with the 2-stage setup method of Benjamini, Krieger, and Yekutieli. For survival experiments, statistical significance was tested by Gehan-Breslow-Wilcoxon test. Correlations were analyzed with Spearman's rank correlation. A $P$ value of less than 0.05 was considered statistically significant. Results are presented as mean \pm SEM, with the exception of human studies for which the median is presented. All experimental values were obtained from the measurement of distinct samples and nonrepeated measures of the same sample. Experiments were repeated at least 2 times, with the exception of CLP and i.p. LPS injection experiments in order to reduce the number of animals used according to the $3 \mathrm{R}$ (replacement, reduction, and refinement) rules and the requirements of the IACUC of the Université de BourgogneFranche-Comté. For microscopy and Western blotting experiments, representative data are presented in the figures.

Study approval. All studies with mice were conducted in accordance with the local guidelines for animal experimentation. Protocol no. 2512 and APAFIS no. 22339-2019100909387172v2 were approved by the IACUC of the Université de Bourgogne-Franche-Comté. All procedures performed in studies involving human participants were carried out in accordance with the ethical standards of the institutional research committee (Comité de Protection des Personnes [CPP], CPP EST I, Dijon, France) and with the 1964 Helsinki Declaration and its later amendments. Human studies were approved by CPP EST I. All enrolled patients 
and/or their next of kin were informed, and written consent authorizing the use of biological samples was documented in the medical records of the patients by the investigator. The observational study Myelochondria is registered under the ClinicalTrials.gov identifier NCT04439617.

General methods statements. No samples, mice, or studies were removed from the analyses, with the exception of unexpected premature death during CLP or i.p. LPS injection experiments. Experiments were not blinded and samples were not randomized in this study. Tissue culture samples were evaluated for mycoplasma contamination.

\section{Author contributions}

CT conceived and supervised experiments, analyzed the data, and wrote the manuscript; LL supervised experiments, wrote the manuscript, and secured funding; DP conceived and performed experiments and analyzed the data; AD and JPQ supervised human studies; M Nguyen, JG, JB, VD, AR, M Narce, MR, DM, and JA provided scientific expertise and critical reading of the manuscript; KVD, FM, VD, AD, AR, AJ, TB, TG, CM, NLG, SM, JB, FD, CS, and NG performed experiments.

\section{Acknowledgments}

We dedicate this work to the memory of Franck Mignotte. The authors acknowledge A. Hamman, S. Monier, N. Pernet (Flow Cytometry Facility); C. Arnould, B. Gasquet (Microscopy and Histology Facilities), E. Charron, H. Choubley, V. Bergas, J.P. Pais De Barros (Lipidomic Facility); V. Saint-Giorgio (Animal Facility); and J. Laurent (UMR PAM) for expert technical expertise. This work was supported by the INSERM (Centre de Recherche UMR 1231), the UBFC, the CHU Dijon, the ANR Investissements d'Avenir Grant (ANR-11 LABX-0021, Labex LipSTIC), the Investissements d'Avenir program (LipoMAC Project, ISITE-BFC, ANR-15-IDEX-0003), the FEDER, and the Regional Council of Bourgogne Franche-Comte and the Ecole Polytechnique Fédérale de Lausanne (EPFL). DP was supported by a fellowship funded by the HEC Pakistan.

Address correspondence to: Charles Thomas, UFR SVTE, Université de Bourgogne, 6 Boulevard Gabriel, 21000 Dijon, France. Phone: 33.3.80.39.63.19; Email: charles.thomas@u-bourgogne.fr.
1. Angus DC, van der Poll T. Severe sepsis and septic shock. N Engl J Med. 2013;369(9):840-851.

2. Nathan C. Points of control in inflammation. Nature. 2002;420(6917):846-852.

3. Cavaillon JM, Eisen D, Annane D. Is boosting the immune system in sepsis appropriate? Crit Care. 2014;18(2):216.

4. Murray PJ, et al. Macrophage activation and polarization: nomenclature and experimental guidelines. Immunity. 2014;41(1):14-20.

5. Hotchkiss RS, Monneret G, Payen D. Sepsis-induced immunosuppression: from cellular dysfunctions to immunotherapy. Nat Rev Immunol. 2013;13(12):862-874.

6. Buck MD, Sowell RT, Kaech SM, Pearce EL. Metabolic instruction of immunity. Cell. 2017;169(4):570-586.

7. O'Neill LA, Kishton RJ, Rathmell J. A guide to immunometabolism for immunologists. Nat Rev Immunol. 2016;16(9):553-565.

8. Mills EL, et al. Succinate dehydrogenase supports metabolic repurposing of mitochondria to drive inflammatory macrophages. Cell. 2016;167(2):457-470.e13.

9. Carchman EH, et al. Experimental sepsis-induced mitochondrial biogenesis is dependent on autophagy, TLR4, and TLR9 signaling in liver. FASEB J. 2013;27(12):4703-4711.

10. Rocheteau P, et al. Sepsis induces long-term metabolic and mitochondrial muscle stem cell dysfunction amenable by mesenchymal stem cell therapy. Nat Commun. 2015;6:10145.

11. Bravo-San Pedro JM, Kroemer G, Galluzzi L. Autophagy and mitophagy in cardiovascular disease. Circ Res. 2017;120(11):1812-1824.

12. Cheng SC, et al. Broad defects in the energy metabolism of leukocytes underlie immunoparalysis in sepsis. Nat Immunol. 2016;17(4):406-413.

13. Kim MJ, et al. SESN2/sestrin2 suppresses sepsis by inducing mitophagy and inhibiting NLRP3 activation in macrophages. Autophagy. 2016;12(8):1272-1291.

14. Zhou R, Yazdi AS, Menu P, Tschopp J. A role for mitochondria in NLRP3 inflammasome activation. Nature. 2011;469(7329):221-225.

15. Nakahira K, et al. Autophagy proteins regulate innate immune responses by inhibiting the release of mitochondrial DNA mediated by the NALP3 inflammasome. Nat Immunol. 2011;12(3):222-230.

16. Arulkumaran N, et al. Mitochondrial function in sepsis. Shock. 2016;45(3):271-281.

17. Youle RJ, Narendra DP. Mechanisms of mitophagy. Nat Rev Mol Cell Biol. 2011;12(1):9-14.

18. Georgakopoulos ND, Wells G, Campanella M. The pharmacological regulation of cellular mitophagy. Nat Chem Biol. 2017;13(2):136-146.

19. Piquereau J, et al. Protective role of PARK2/ Parkin in sepsis-induced cardiac contractile and mitochondrial dysfunction. Autophagy. 2013;9(11):1837-1851.

20. Thomas KJ, et al. DJ-1 acts in parallel to the PINK1/parkin pathway to control mitochondrial function and autophagy. Hum Mol Genet. 2011;20(1):40-50.

21. Xiong $\mathrm{H}$, et al. Parkin, PINK1, and DJ-1 form a ubiquitin E3 ligase complex promoting unfolded protein degradation. J Clin Invest. 2009;119(3):650-660.

22. Amatullah H, et al. DJ-1/PARK7 impairs bacterial clearance in sepsis. Am J Respir Crit Care Med. 2017;195(7):889-905

23. Nathan C, Cunningham-Bussel A. Beyond oxidative stress: an immunologist's guide to reactive oxygen species. Nat Rev Immunol. 2013;13(5):349-361.

24. Weinberg SE, Sena LA, Chandel NS. Mitochondria in the regulation of innate and adaptive immunity. Immunity. 2015;42(3):406-417.

25. Garaude J, et al. Mitochondrial respiratory-chain adaptations in macrophages contribute to antibacterial host defense. Nat Immunol. 2016;17(9):1037-1045.

26. West AP, et al. TLR signalling augments macrophage bactericidal activity through mitochondrial ROS. Nature. 2011;472(7344):476-480.
27. Geng J, et al. Kinases Mst1 and Mst2 positively regulate phagocytic induction of reactive oxygen species and bactericidal activity. Nat Immunol. 2015;16(11):1142-1152.

28. Andreux PA, Houtkooper RH, Auwerx J. Pharmacological approaches to restore mitochondrial function. Nat Rev Drug Discov. 2013;12(6):465-483.

29. Xu Y, Jagannath C, Liu XD, Sharafkhaneh A, Kolodziejska KE, Eissa NT. Toll-like receptor 4 is a sensor for autophagy associated with innate immunity. Immunity. 2007;27(1):135-144.

30. Dodson MW, Guo M. Pink1, Parkin, DJ-1 and mitochondrial dysfunction in Parkinson's disease. Curr Opin Neurobiol. 2007;17(3):331-337.

31. Pickles S, Vigié P, Youle RJ. Mitophagy and quality control mechanisms in mitochondrial maintenance. Curr Biol. 2018;28(4):R170-R185.

32. Lachmandas E, et al. Microbial stimulation of different Toll-like receptor signalling pathways induces diverse metabolic programmes in human monocytes. Nat Microbiol. 2016;2:16246.

33. Gomes LC, Di Benedetto G, Scorrano L. During autophagy mitochondria elongate, are spared from degradation and sustain cell viability. Nat Cell Biol. 2011;13(5):589-598.

34. Khacho M, et al. Acidosis overrides oxygen deprivation to maintain mitochondrial function and cell survival. Nat Commun. 2014;5:3550.

35. Merx MW, et al. HMG-CoA reductase inhibitor simvastatin profoundly improves survival in a murine model of sepsis. Circulation. 2004;109(21):2560-2565.

36. Venet F, Lepape A, Monneret G. Clinical review: flow cytometry perspectives in the ICU - from diagnosis of infection to monitoring of injuryinduced immune dysfunctions. Crit Care. 2011;15(5):231.

37. Yu J, et al. Inflammasome activation leads to Caspase-1-dependent mitochondrial damage and block of mitophagy. Proc Natl Acad Sci USA. 2014;111(43):15514-15519.

38. Kahns S, Kalai M, Jakobsen LD, Clark BF, Vandenabeele P, Jensen PH. Caspase-1 and 
caspase-8 cleave and inactivate cellular parkin. J Biol Chem. 2003;278(26):23376-23380.

39. Chin YE, Kitagawa M, Kuida K, Flavell RA, Fu XY. Activation of the STAT signaling pathway can cause expression of caspase 1 and apoptosis. Mol Cell Biol. 1997;17(9):5328-5337.

40. Man SM, Kanneganti TD. Converging roles of caspases in inflammasome activation, cell death and innate immunity. Nat Rev Immunol. 2016;16(1):7-21.

41. Kayagaki N, et al. Non-canonical inflammasome activation targets caspase-11. Nature. 2011;479(7371):117-121.

42. Py BF, et al. Caspase-11 controls interleukin-1 $\beta$ release through degradation of TRPC1. Cell Rep. 2014;6(6):1122-1128.

43. Woodroof HI, et al. Discovery of catalytically active orthologues of the Parkinson's disease kinase PINK1: analysis of substrate specificity and impact of mutations. Open Biol. 2011;1(3):110012.

44. Kane LA, et al. PINK1 phosphorylates ubiquitin to activate Parkin E3 ubiquitin ligase activity. J Cell Biol. 2014;205(2):143-153.

45. Wauer T, Simicek M, Schubert A, Komander D. Mechanism of phospho-ubiquitin-induced PARKIN activation. Nature. 2015;524(7565):370-374.

46. Hagar JA, Powell DA, Aachoui Y, Ernst RK, Miao EA. Cytoplasmic LPS activates caspase-11: implications in TLR4-independent endotoxic shock. Science. 2013;341(6151):1250-1253.

47. Shi J, et al. Inflammatory caspases are innate immune receptors for intracellular LPS. Nature. 2014;514(7521):187-192.

48. Chandel NS, et al. Reactive oxygen species generated at mitochondrial complex III stabilize hypoxia-inducible factor-1alpha during hypoxia: a mechanism of $\mathrm{O} 2$ sensing. J Biol Chem. 2000;275(33):25130-25138.

49. Shalova IN, et al. Human monocytes undergo functional re-programming during sepsis mediated by hypoxia-inducible factor-1 $\alpha$. Immunity . 2015;42(3):484-498.

50. Cheng SC, et al. mTOR- and HIF-1 $\alpha$-mediated aerobic glycolysis as metabolic basis for trained immunity. Science. 2014;345(6204):1250684.

51. Tannahill GM, et al. Succinate is an inflammatory signal that induces IL-1 $\beta$ through HIF- $1 \alpha$. Nature. 2013;496(7444):238-242.

52. Kong D, et al. Echinomycin, a small-molecule inhibitor of hypoxia-inducible factor-1 DNA-binding activity. Cancer Res. 2005;65(19):9047-9055.

53. Singer M, et al. The third international consensus definitions for sepsis and septic shock (sepsis-3). JAMA. 2016;315(8):801-810.

54. Zhong Z, et al. NF-kB restricts inflammasome activation via elimination of damaged mitochondria. Cell. 2016;164(5):896-910.

55. Rademann P, et al. Mitochondria-targeted antioxidants SkQ1 and MitoTEMPO failed to exert a long-term beneficial effect in murine polymicrobial sepsis. Oxid Med Cell Longev. 2017;2017:6412682.

56. Ghanta S, et al. Mesenchymal stromal cells deficient in autophagy proteins are susceptible to oxidative injury and mitochondrial dysfunction. Am J Respir Cell Mol Biol. 2017;56(3):300-309.

57. Mannam P, et al. MKK3 regulates mitochondrial biogenesis and mitophagy in sepsis-induced lung injury. Am J Physiol Lung Cell Mol Physiol. 2014;306(7):L604-L619.

58. Rambold AS, Pearce EL. Mitochondrial dynamics at the interface of immune cell metabolism and function. Trends Immunol. 2018;39(1):6-18.

59. Liu W, Acín-Peréz R, Geghman KD, Manfredi G, Lu B, Li C. Pink1 regulates the oxidative phosphorylation machinery via mitochondrial fission. Proc Natl Acad Sci USA. 2011;108(31):12920-12924.

60. Requejo-Aguilar R, Lopez-Fabuel I, Fernandez E, Martins LM, Almeida A, Bolaños JP. PINK1 deficiency sustains cell proliferation by reprogramming glucose metabolism through HIF1. Nat Commun. 2014;5:4514.

61. Yao Z, Gandhi S, Burchell VS, Plun-Favreau H, Wood NW, Abramov AY. Cell metabolism affects selective vulnerability in PINK1-associated Parkinson's disease. JCell Sci. 2011;124(Pt 24):4194-4202. 62. Sica A, Mantovani A. Macrophage plasticity and polarization: in vivo veritas. JClin Invest. 2012;122(3):787-795.

63. Bourke LT, Knight RA, Latchman DS, Stephanou A, McCormick J. Signal transducer and activator of transcription-1 localizes to the mitochondria and modulates mitophagy. JAKSTAT. 2013;2(4):e25666.

64. Pitroda SP, et al. STAT1-dependent expression of energy metabolic pathways links tumour growth and radioresistance to the Warburg effect. $B M C$ Med. 2009;7:68.

65. Sisler JD, et al. The signal transducer and activator of transcription 1 (STAT1) inhibits mitochondrial biogenesis in liver and fatty acid oxidation in adipocytes. PLOS ONE. 2015;10(12):e0144444.

66. Broz P, Dixit VM. Inflammasomes: mechanism of assembly, regulation and signalling. Nat Rev Immunol. 2016;16(7):407-420.

67. Dargent A, et al. Improved quantification of plasma lipopolysaccharide (LPS) burden in sepsis using 3-hydroxy myristate (3HM): a cohort study. Intensive Care Med. 2019;45(11):1678-1680.

68. Moullan N, et al. Tetracyclines disturb mitochondrial function across eukaryotic models: a call for caution in biomedical research. Cell Rep. 2015;10(10):1681-1691.

69. Katayama H, Kogure T, Mizushima N, Yoshimori T, Miyawaki A. A sensitive and quantitative technique for detecting autophagic events based on lysosomal delivery. Chem Biol. 2011;18(8):1042-1052.

70. Bastin J, Aubey F, Rötig A, Munnich A, Djouadi F. Activation of peroxisome proliferator-activated receptor pathway stimulates the mitochondrial respiratory chain and can correct deficiencies in patients' cells lacking its components. J Clin Endocrinol Metab. 2008;93(4):1433-1441.

71. Deckert V, et al. Recombinant human plasma phospholipid transfer protein (PLTP) to prevent bacterial growth and to treat sepsis. Sci Rep. 2017;7(1):3053.

72. De Felice FG, Ferreira ST. Novel neuroprotective, neuritogenic and anti-amyloidogenic properties of 2,4-dinitrophenol: the gentle face of Janus. IUBMB Life. 2006;58(4):185-191. 